\title{
Integral inequalities for a fractional operator of a function with respect to another function with nonsingular kernel
}

\author{
Pshtiwan Othman Mohammed ${ }^{1}$ (D) and Thabet Abdeljawad ${ }^{2,3,4^{*}}$ (D)
}

\section{"Correspondence:}

tabdeljawad@psu.edu.sa

${ }^{2}$ Department of Mathematics and

General Sciences, Prince Sultan

University, P.O. Box 66833, Riyadh

11586, Saudi Arabia

${ }^{3}$ Department of Medical Research,

China Medical University, Taichung

40402, Taiwan

Full list of author information is

available at the end of the article

\section{Springer}

\begin{abstract}
At first, we construct a connection between the Atangana-Baleanu and the Riemann-Liouville fractional integrals of a function with respect to a monotone function with nonsingular kernel. By examining this relationship and the iterated form of Prabhakar fractional model, we are able to find some new Hermite-Hadamard inequalities and related results on integral inequalities for the two models of fractional calculus which are defined using monotone functions with nonsingular kernels.
\end{abstract}

MSC: 26D07; 26D10;26D15;26A33

Keywords: Riemann-Liouville fractional integral; Mittag-Leffler function; Integral inequalities

\section{Introduction}

As we know, the fractional calculus is a fundamental model considered as a powerful tool in many fields, for example, rheology, dynamic systems, biophysics, electrical networks, blood flow phenomena; see for details [6, 32, 43, 52]. On September 1695 the fractional calculus was considered by Leibniz as a new model of mathematical analysis which involves derivatives and integrals taken to fractional orders or orders outside of the integer numbers. After Leibniz, many famous mathematicians and physicians have studied theory and application of this model for fractional derivatives and integrals and fractional differential equations; see for detail [12, 13, 35, 55]. Some of them were Grunwald, Liouville, Riemann-Letnikov, Caputo, and Atangana-Baleanu which we will consider in more detail in the next section. The inequality is a modern model of mathematical analysis that describes the growth rate of competing mathematical analysis. This model is also used in various fields such as integral equations, ordinary and partial differential equations [29, 59]. In particular, they have been successfully used in the study of fractional differential equations and sometimes called fractional integral inequalities. They have been vital in proving the uniqueness or existence of solutions for some well-known fractional differential equations and in providing boundedness to solve certain fractional boundary and initial value problems, see for detail $[1,7,19,24,26,33,34,41,48-51]$. The rest of the article is organized as follows: In Sect. 2, we recall some basic definitions and tools about fractional derivatives

(c) The Author(s) 2020. This article is licensed under a Creative Commons Attribution 4.0 International License, which permits use sharing, adaptation, distribution and reproduction in any medium or format, as long as you give appropriate credit to the original author(s) and the source, provide a link to the Creative Commons licence, and indicate if changes were made. The images or other third party material in this article are included in the article's Creative Commons licence, unless indicated otherwise in a credit line to the material. If material is not included in the article's Creative Commons licence and your intended use is not permitted by statutory regulation or exceeds the permitted use, you will need to obtain permission directly from the copyright holder. To view a copy of this licence, visit http://creativecommons.org/licenses/by/4.0/. 
with Mittag-Leffler and power law kernels of a function with respect to another function in the sense of Caputo and Riemann and their corresponding integrals. The existence of the strictly increasing function in the kernel allows us to include a large class of fractional operators, and hence our results will generalize and unify many existing results in the literature treating Hermite-Hadamard inequalities. We start Sect. 3 by introducing the corresponding fractional integral of the fractional operators with Mittag-Leffler kernels depending on a function and proving it for the first time. Then, we proceed to proving the Hermite-Hadamard fractional inequalities in the setting of these generalized fractional integrals. An example with supporting figures is presented in this section as well to clarify the proved main result. We devote Sect. 4 to presenting a related trapezoidal equality for such a class of fractional integrals. Finally, in Sect. 5, we outline our conclusions about the novel results we have proved in this short interesting study.

\section{Definitions and preliminaries}

Always, it is important and necessary to specify which model or definition is being used because there are many different ways of defining fractional integrals and derivatives. The most frequent definition of fractional integrals and derivatives is the Riemann-Liouville one, in which fractional integrals and derivatives are defined as follows.

Definition 2.1 ([32, 43, 52]) For any $\mathrm{L}^{1}$ function $f(x)$ on an interval $\left[v_{1}, v_{2}\right]$ with $x \in$ $\left[v_{1}, v_{2}\right]$, the $\eta_{1}$ th left-RL fractional integral of $f(x)$ is defined by

$$
{ }^{R L} \mathcal{J}_{\nu_{1}}^{\eta_{1}} f(x):=\frac{1}{\Gamma\left(\eta_{1}\right)} \int_{v_{1}}^{x}(x-\xi)^{\eta_{1}-1} f(\xi) \mathrm{d} \xi
$$

for $\operatorname{Re}\left(\eta_{1}\right)>0$. Also, for any $C^{n}$ function $f(x)$ on an interval $\left[v_{1}, v_{2}\right]$ with $x \in\left[v_{1}, v_{2}\right]$, the $\eta_{1}$ th left-RL fractional derivative of $f(x)$ is defined by

$$
{ }^{R L} \mathrm{D}_{\nu_{1}+}^{\eta_{1}} f(x):=\frac{\mathrm{d}^{n}}{\mathrm{~d} x^{n}} R L \mathrm{~J}_{\nu_{1}+}^{n-\eta_{1}} f(x)
$$

for $n-1 \leq \operatorname{Re}\left(\eta_{1}\right)<n$.

Observe that since ${ }^{R L} \mathrm{D}_{v_{1}+}^{-\eta_{1}} f(x)={ }^{R L} \mathrm{~J}_{v_{1}+}^{\eta_{1}} f(x)$ and the derivative formula (2) is the analytic continuation of the integral formula (1) in $\eta_{1}$, thus differentiation and integration can be unified in a single operator that we call differintegration.

Definition 2.2 ([52]) For any $\mathrm{L}^{1}$ function $f(x)$ on an interval $\left[v_{1}, v_{2}\right]$ with $x \in\left[v_{1}, v_{2}\right]$, the $\eta_{1}$ th right-RL fractional integral of $f(x)$ is defined by

$$
R L_{v_{v_{2}}}^{\eta_{1}} f(x):=\frac{1}{\Gamma\left(\eta_{1}\right)} \int_{x}^{v_{2}}(\xi-x)^{\eta_{1}-1} f(\xi) \mathrm{d} \xi
$$

for $\operatorname{Re}\left(\eta_{1}\right)>0$. Also, for any $C^{n}$ function $f(x)$ on an interval $\left[v_{1}, v_{2}\right]$ with $x \in\left[v_{1}, v_{2}\right]$, the $\eta_{1}$ th right-RL fractional derivative of $f(x)$ is defined by

$$
{ }^{R L} \mathrm{D}_{v_{2}-}^{\eta_{1}} f(x):=(-1)^{n} \frac{\mathrm{d}^{n}}{\mathrm{~d} x^{n}}{ }^{R L} \mathrm{~J}_{v_{2}-}^{n-\eta_{1}} f(x)
$$

for $n-1 \leq \operatorname{Re}\left(\eta_{1}\right)<n$. 
In the recent decades, a strong modern direction of research in fractional calculus has brought the attention of interested researchers in various disciplines to investigate various possible ways to define fractional integrals and derivatives, often with different properties from the classical RL definition. Some of them are more effective than the RL model that can be used to model real-life data $[8,23]$. One of the most recent models of fractional calculus discussed in this paper is the fractional calculus of a function with respect to another function (which is often called $\psi$-RL fractional calculus) that was firstly defined in the classical RL model by Osler [44] and the concept has been extended by many authors; e.g. $[17,56]$. As shown in many papers cited below, it has been especially useful in realworld modeling.

Definition $2.3([17,44,56])$ Suppose that $\left(v_{1}, v_{2}\right) \subseteq \mathfrak{R}$ is a finite or infinite interval of the real numbers $\mathfrak{R}$ and $\psi(x)$ is an increasing positive monotone function on the interval $\left[v_{1}, v_{2}\right]$ with $\psi^{\prime}(x) \in \mathrm{L}^{1}\left(v_{1}, v_{2}\right)$. Then the $\psi$-RL fractional integrals of a function $f$ with respect to another function $\psi(x)$ on $\left[v_{1}, v_{2}\right]$ is defined by

$$
\mathrm{J}_{v_{1}+}^{\eta_{1}} f(x)=\frac{1}{\Gamma\left(\eta_{1}\right)} \int_{v_{1}}^{x} \psi^{\prime}(\xi)(\psi(x)-\psi(\xi))^{\eta_{1}-1} f(\xi) \mathrm{d} \xi
$$

for $\eta_{1} \in \mathbb{C}$ and $\operatorname{Re}\left(\eta_{1}\right)>0$. Furthermore, the $\psi$ - $\mathrm{R}$ fractional derivatives of a function $f$ with respect to another function $\psi(x)$ is defined by

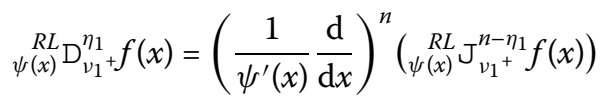

for $\eta_{1} \in \mathbb{C}$ with $\operatorname{Re}\left(\eta_{1}\right) \geq 0$ and $n-1 \leq \operatorname{Re}\left(\eta_{1}\right)<n$.

Special functions have a strong relationship with fractional calculus $[28,32]$, and the Mittag-Leffler function is a particular important one in this area, see e.g. [21, 22, 31]. There are two important models of fractional calculus which have been defined by integrals similar to (1) but with Mittag-Leffler (ML) functions in the kernel, namely the Atangana-Baleanu and Prabhakar models $[9,10]$.

Definition 2.4 ([17]) For any function $f(x)$ which is differentiable with $f^{\prime} \in \mathrm{L}^{1}\left[v_{1}, v_{2}\right]$ and $x \in\left[v_{1}, v_{2}\right]$, the $\eta_{1}$ th Atangana-Baleanu (AB) fractional derivative of $f(x)$ with respect to another function $\psi(x)$ in the RL sense is defined by

$$
{ }_{\psi(x)}^{A B R} D_{\nu_{1}+}^{\eta_{1}} f(x):=\frac{B\left(\eta_{1}\right)}{\left(1-\eta_{1}\right) \psi^{\prime}(x)} \cdot \frac{\mathrm{d}}{\mathrm{d} x} \int_{\nu_{1}}^{x} \psi^{\prime}(\xi) E_{\eta_{1}}\left(\frac{-\eta_{1}}{1-\eta_{1}}(\psi(x)-\psi(\xi))^{\eta_{1}}\right) f(\xi) \mathrm{d} \xi,
$$

and in the Caputo sense it is defined by

$$
\nu_{\psi(x)}^{A B R} D_{\nu_{1}+}^{\eta_{1}} f(x):=\frac{B\left(\eta_{1}\right)}{1-\eta_{1}} \int_{\nu_{1}}^{x} E_{\eta_{1}}\left(\frac{-\eta_{1}}{1-\eta_{1}}(\psi(x)-\psi(\xi))^{\eta_{1}}\right) f^{\prime}(\xi) \mathrm{d} \xi
$$

for $0<\eta_{1}<1$, where $\psi(x)$ is as before, $E_{\eta_{1}}(z)$ is the standard one-parameter ML function, and $B\left(\eta_{1}\right)>0$ is a normalization function that satisfies $B(0)=B(1)=1$.

It is clear that the above definitions are for left $\mathrm{AB}$ integrals and derivatives. In a natural way similar to Definition 2.2, we can define right $\mathrm{AB}$ integrals and derivatives possibly. 
Definition $2.5([27,46])$ For any $\mathrm{L}^{1}$ function $f(x)$ on an interval $\left[v_{1}, v_{2}\right]$ with $x \in\left[v_{1}, v_{2}\right]$, the Prabhakar fractional integral operator is defined by

$$
{ }^{P} \mathrm{~J}_{v_{1}+}^{\eta_{1}, \eta_{2}, \gamma, \omega} f(x):=\int_{v_{1}}^{x}(x-\xi)^{\eta_{2}-1} E_{\eta_{1}, \eta_{2}}^{\gamma}\left(\omega(x-\xi)^{\eta_{1}}\right) f(\xi) \mathrm{d} \xi
$$

for $\operatorname{Re}\left(\eta_{1}\right)>0, \operatorname{Re}\left(\eta_{2}\right)>0$, and $\gamma, \omega \in \mathbb{C}$, where $E_{\eta_{1}, \eta_{2}}^{\gamma}(z)$ is the three-parameter ML function.

Also, for any $C^{n}$ function $f(x)$ on an interval $\left[v_{1}, v_{2}\right]$ with $x \in\left[v_{1}, v_{2}\right]$, the Prabhakar fractional derivative operator is defined as follows:

$$
{ }^{P} \mathrm{D}_{\nu_{1}+}^{\eta_{1}, \eta_{2}, \gamma, \omega} f(x):=\frac{\mathrm{d}^{n}}{\mathrm{~d} x^{n}}\left({ }^{P} \mathrm{~J}_{\nu_{1}+}^{\eta_{1}, n-\eta_{2},-\gamma, \omega} f(x)\right)
$$

for $\operatorname{Re}\left(\eta_{1}\right)>0, n-1 \leq \operatorname{Re}\left(\eta_{2}\right)<n$, and $\gamma, \omega \in \mathbb{C}$.

Again, the above definitions are for left Prabhakar integrals and derivatives. In a natural way similar to Definition 2.2, we can define right Prabhakar integrals and derivatives possibly.

Remark 2.1 Observe that Definition 2.5 can be seen as a special case of the generalized Prabhakar model defined in [57].

Definition 2.6 ([17]) For any $\mathrm{L}^{1}$ function $f(x)$ on an interval $\left[v_{1}, v_{2}\right]$ with $x \in\left[v_{1}, v_{2}\right]$, the generalized Prabhakar fractional integral operator of a function $f(x)$ with respect to another function $\psi(x)$ is defined by

$$
\underset{\psi(x)}{P} \mathrm{~J}_{\nu_{1}+}^{\eta_{1}, \eta_{2}, \gamma, \omega} f(x):=\int_{\nu_{1}}^{x}(\psi(x)-\psi(\xi))^{\eta_{2}-1} E_{\eta_{1}, \eta_{2}}^{\gamma, \kappa}\left(\omega(\psi(x)-\psi(\xi))^{\eta_{1}}\right) f(\xi) \mathrm{d} \xi
$$

for $\operatorname{Re}\left(\eta_{1}\right)>0, \operatorname{Re}\left(\eta_{2}\right)>0, \operatorname{Re}(\kappa)>0, \operatorname{Re}\left(\kappa-\eta_{1}\right)<1$, and $\gamma, \omega \in \mathbb{C}$, where $\psi(x)$ is as before and $E_{\eta_{1}, \eta_{2}}^{\gamma, \kappa}(z)$ is the generalized ML function defined by

$$
E_{\eta_{1}, \eta_{2}}^{\gamma, \kappa}(z):=\sum_{n=0}^{\infty} \frac{\Gamma(\gamma+\kappa n) z^{n}}{\Gamma(\gamma) \Gamma\left(\eta_{1} n+\eta_{2}\right)}
$$

Also, for any $C^{n}$ function $f(x)$ on an interval $\left[v_{1}, v_{2}\right]$ with $x \in\left[v_{1}, v_{2}\right]$, the generalized Prabhakar fractional derivative operator of a function $f(x)$ with respect to another function $\psi(x)$ is defined by

$$
\underset{\psi(x)}{P} D_{\nu_{1}+}^{\eta_{1}, \eta_{2}, \gamma, \omega} f(x):=\frac{\mathrm{d}^{n}}{\mathrm{~d} x^{n}}\left(\underset{\psi(x)}{P} \mathrm{~J}_{\nu_{1}+}^{\eta_{1}, n-\eta_{2},-\gamma, \omega} f(x)\right)
$$

for $\operatorname{Re}\left(\eta_{1}\right)>0$ and $n-1 \leq \operatorname{Re}\left(\eta_{2}\right)<n$ and $\gamma, \omega \in \mathbb{C}$.

It is important to see Definitions 2.4 and 2.6 as interesting analogues of the basic Definition 2.1 from a pure mathematical point of view. Also, they have their own properties consistent with those of the ML functions [5, 15, 18, 27]. On the other hand, if we look carefully from an applied science point of view, we can deduce that these models of fractional calculus can be matched with real data where power law behavior disappears $[14,20,58]$. 
In the current study, we point out the uniting topic of integral inequalities involving the aforementioned novel models of fractional calculus. Particularly, we consider the wellknown Hermite-Hadamard $(\mathrm{HH})$ inequality, which has been successfully studied for RL fractional integrals. Also, we prove some new results analogous to the classical results which are valid in the generalized $\mathrm{AB}$ and Prabhakar models of fractional calculus. Finally, a related inequality of trapezoidal type is pointed out.

\section{The new fractional $\mathrm{HH}$-inequality}

Our next findings are based on the following lemma.

Lemma 3.1 For any $\mathrm{L}^{1}$ function $f(x)$ on an interval $\left[v_{1}, v_{2}\right]$ with $x \in\left[v_{1}, v_{2}\right]$, the $\eta_{1}$ th Atangana-Baleanu $(A B)$ fractional integral of a function $f(x)$ with respect to another function can be represented as follows:

$$
\underset{\psi(x)}{A B} \mathrm{~J}_{\nu_{1}+}^{\eta_{1}} f(x):=\frac{\eta_{1}}{B\left(\eta_{1}\right)} \underset{\psi(x)}{R L} \mathrm{~J}_{\nu_{1}+}^{\eta_{1}} f(x)+\frac{1-\eta_{1}}{B\left(\eta_{1}\right)} f(x), \quad 0<\eta_{1}<1,
$$

where $B\left(\eta_{1}\right)$ and $\psi(x)$ are as before.

Proof The expression (14) is well defined iff the $\psi$-RL integral $\underset{\psi(x)}{R L} \mathrm{~J}_{\nu_{1}+}^{\eta_{1}} f(x)$ is well defined, which matches our assumptions on $f$ for the $\eta_{1}$ th $\psi$-ABR fractional derivative to be well defined. Following [17] where the AB fractional derivatives of a function $f(x)$ with respect to another function $\psi(x)$ can be represented in the series form

$$
{ }_{\psi(x)}^{A B R} \mathrm{D}_{\nu_{1}+}^{\eta_{1}} f(x)=\frac{B\left(\eta_{1}\right)}{1-\eta_{1}} \sum_{n=0}^{\infty}\left(\frac{-\eta_{1}}{1-\eta_{1}}\right)^{n} \underset{\psi(x)}{R L} \mathrm{~J}_{\nu_{1}+}^{n \eta_{1}} f(x) .
$$

It is sufficient if we show that

$$
{ }_{\psi(x)}^{A B R} D_{\nu_{1}+}^{\eta_{1}}\left(\begin{array}{c}
A B \\
\psi(x) \\
J_{\nu_{1}+}^{\eta_{1}}
\end{array}(x)\right)=f(x)
$$

and

$$
\underset{\psi(x)}{A B} \mathrm{~J}_{v_{1}+}^{\eta_{1}}\left(\begin{array}{l}
A B R \\
\psi(x)
\end{array} D_{v_{1}+}^{\eta_{1}} f(x)\right)=f(x)
$$

for each $a, \eta_{1}, f, \psi$ as stated. By using (15), we deduce that

$$
\begin{aligned}
& { }_{\psi(x)}^{A B R} D_{v_{1}+}^{\eta_{1}}\left(\begin{array}{c}
A B \\
\psi(x)
\end{array} \mathrm{J}_{v_{1}+}^{\eta_{1}} f(x)\right) \\
& ={ }_{\psi(x)}^{A B R} D_{\nu_{1}+}^{\eta_{1}}\left(\frac{\eta_{1}}{B\left(\eta_{1}\right)} \underset{\psi(x)}{R L} \mathrm{~J}_{v_{1}+}^{\eta_{1}} f(x)+\frac{1-\eta_{1}}{B\left(\eta_{1}\right)} f(x)\right) \\
& =\frac{\eta_{1}}{B\left(\eta_{1}\right)} \underset{\psi(x)}{A R} D_{\nu_{1+}}^{\eta_{1}}\left(\begin{array}{r}
R L \\
\psi(x)
\end{array} J_{\nu_{1+}}^{\eta_{1}} f(x)\right)+\frac{1-\eta_{1}}{B\left(\eta_{1}\right)} \psi(x) D_{\nu_{1}+}^{\eta_{1}} f(x) \\
& =\frac{\eta_{1}}{1-\eta_{1}} \sum_{n=0}^{\infty}\left(\frac{-\eta_{1}}{1-\eta_{1}}\right)^{n} \underset{\psi(x)}{R L} \mathrm{~J}_{\nu_{1}+}^{n \eta_{1}}\left(\begin{array}{c}
R L \\
\psi(x)
\end{array} \mathrm{J}_{\nu_{1}+}^{\eta_{1}} f(x)\right) \\
& +\sum_{n=0}^{\infty}\left(\frac{-\eta_{1}}{1-\eta_{1}}\right)^{n} \underset{\psi(x)}{R L} \mathrm{~J}_{v_{1}+}^{n \eta_{1}} f(x)
\end{aligned}
$$




$$
\begin{aligned}
& =\sum_{n=0}^{\infty}\left(\frac{-\eta_{1}}{1-\eta_{1}}\right)^{n} \underset{\psi(x)}{R L} \mathrm{~J}_{v_{1}+}^{n \eta_{1}} f(x)-\sum_{n=0}^{\infty}\left(\frac{-\eta_{1}}{1-\eta_{1}}\right)^{n+1} \underset{\psi(x)}{R L} \mathrm{~J}_{v_{1}+}^{n \eta_{1}+\eta_{1}} f(x) \\
& =f(x) \text {; } \\
& \underset{\psi(x)}{A B} \mathrm{~J}_{v_{1}+}^{\eta_{1}}\left(\begin{array}{l}
A B R(x) \\
\psi\left(v_{1}+\right.
\end{array} D^{\eta_{1}} f(x)\right)
\end{aligned}
$$

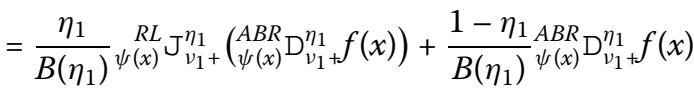

$$
\begin{aligned}
& =\frac{\eta_{1}}{1-\eta_{1}} \psi(x) \mathrm{J}_{v_{1}+}^{\eta_{1}}\left(\sum_{n=0}^{\infty}\left(\frac{-\eta_{1}}{1-\eta_{1}}\right)^{n} \underset{\psi(x)}{R L} \mathrm{~J}_{v_{1}+}^{n \eta_{1}} f(x)\right) \\
& +\sum_{n=0}^{\infty}\left(\frac{-\eta_{1}}{1-\eta_{1}}\right)^{n} \underset{\psi(x)}{R L} \mathrm{~J}_{v_{1}+}^{n \eta_{1}} f(x) \\
& =\sum_{n=0}^{\infty}\left(\frac{-\eta_{1}}{1-\eta_{1}}\right)^{n} \underset{\psi(x)}{R L} \mathrm{~J}_{v_{1}}^{n \eta_{1}} f(x)-\sum_{n=0}^{\infty}\left(\frac{-\eta_{1}}{1-\eta_{1}}\right)^{n+1} \stackrel{R L}{R(x)} \mathrm{J}_{v_{1}+}^{n \eta_{1}+\eta_{1}} f(x) \\
& =f(x) \text {; }
\end{aligned}
$$

which completes the proof.

Remark 3.1 The discrete version of (15) was obtained in [4] and its generalization to the case of generalized ML function case together with its discrete counterpart can be found in [2] and [3], respectively.

Now, we recall the standard Hermite-Hadamard $(\mathrm{HH})$ inequality for an $\mathrm{L}^{1}$ convex function $f:\left[v_{1}, v_{2}\right] \rightarrow \mathfrak{R}$ as follows:

$$
f\left(\frac{v_{1}+v_{2}}{2}\right) \leq \frac{1}{v_{2}-v_{1}} \int_{v_{1}}^{v_{2}} f(x) \mathrm{d} x \leq \frac{f\left(v_{1}\right)+f\left(v_{2}\right)}{2} .
$$

In 2013, HH-inequality (16) was generalized to fractional integrals of RL type by Sarikaya et al. [53], which is as follows:

$$
f\left(\frac{v_{1}+v_{2}}{2}\right) \leq \frac{\Gamma\left(\eta_{1}+1\right)}{2\left(v_{2}-v_{1}\right)^{\eta_{1}}}\left[{ }^{R L} \mathrm{~J}_{v_{1}+}^{\eta_{1}} f\left(v_{2}\right)+{ }^{R L} \mathrm{~J}_{v_{2}-}^{\eta_{1}} f\left(v_{1}\right)\right] \leq \frac{f\left(v_{1}\right)+f\left(v_{2}\right)}{2},
$$

where $f$ is as before and $\eta_{1}>0$.

Most recently, in 2019, $\mathrm{HH}$-inequality (16) was generalized to fractional integrals of $\psi$ RL type by Liu et al. [30] for an $\mathrm{L}^{1}$ convex and positive function $f:\left[v_{1}, v_{2}\right] \rightarrow \mathfrak{R}$ and for an increasing positive monotone function $\psi$ on $\left(v_{1}, v_{2}\right]$ with $\psi^{\prime}(x) \in \mathrm{L}^{1}\left(v_{1}, v_{2}\right)$ :

$$
\begin{aligned}
& f\left(\frac{\nu_{1}+v_{2}}{2}\right) \\
& \leq \frac{\Gamma\left(\eta_{1}+1\right)}{2\left(v_{2}-v_{1}\right)^{\eta_{1}}}\left[{ }_{\psi(x)}^{R L} \mathrm{~J}_{\psi^{-1}\left(v_{1}\right)+}^{\eta_{1}}(f \circ \psi)\left(\psi^{-1}\left(v_{2}\right)\right)+\underset{\psi(x)}{R L} 丁_{\psi^{-1}\left(v_{2}\right)-}^{\eta_{1}}(f \circ \psi)\left(\psi^{-1}\left(v_{1}\right)\right)\right] \\
& \leq \frac{f\left(v_{1}\right)+f\left(v_{2}\right)}{2}, \quad \eta_{1}>0 .
\end{aligned}
$$

Many further results have been derived from inequality (17); for details, see [16, 25, 34, 38, 47]. But so far such inequalities have not been investigated for other models of fractional 
calculus involving Mittag-Leffler kernels. We do so here, presenting the main results in the following three theorems.

Theorem 3.1 Iff $:\left[v_{1}, v_{2}\right] \rightarrow \mathfrak{R}$ is an $\mathrm{L}^{1}$ convex function, $\psi$ is an increasing positive function on $\left(v_{1}, v_{2}\right]$ with $\psi^{\prime}(x) \in \mathrm{L}^{1}\left(v_{1}, v_{2}\right)$, and $\eta_{1} \in(0,1)$, then we have

$$
\begin{aligned}
& f\left(\frac{\nu_{1}+v_{2}}{2}\right) \leq \frac{B\left(\eta_{1}\right) \Gamma\left(\eta_{1}\right)}{2\left[\left(v_{2}-v_{1}\right)^{\eta_{1}}+\left(1-\eta_{1}\right) \Gamma\left(\eta_{1}\right)\right]}[\underset{\psi(x)}{A B})_{\psi^{-1}\left(\nu_{1}\right)+}^{\eta_{1}}(f \circ \psi)\left(\psi^{-1}\left(v_{2}\right)\right) \\
& \left.+\underset{\psi(x)}{A B} \mathrm{~J}_{\psi^{-1}\left(\nu_{2}\right)-}^{\eta_{1}}(f \circ \psi)\left(\psi^{-1}\left(v_{1}\right)\right)\right] \\
& \leq \frac{f\left(v_{1}\right)+f\left(v_{2}\right)}{2} \text {. }
\end{aligned}
$$

Proof From (14), we see that the $\mathrm{AB}$ fractional integral of a function $f(x)$ is clearly a linear combination of $f(x)$ itself with its $\psi$-RL fractional integral. Then we have

$$
\begin{aligned}
& \underset{\psi(x)}{A B} \mathrm{~J}_{\psi^{-1}\left(v_{1}\right)_{+}}^{\eta_{1}}(f \circ \psi)\left(\psi^{-1}\left(v_{2}\right)\right)+\underset{\psi(x)}{A B} \mathrm{~J}_{\psi^{-1}\left(v_{2}\right)_{-}}^{\eta_{1}}(f \circ \psi)\left(\psi^{-1}\left(v_{1}\right)\right) \\
& =\left[\frac{\eta_{1}}{B\left(\eta_{1}\right)} \underset{\psi(x)}{R L} \mathrm{~J}_{\psi^{-1}\left(v_{1}\right)+}^{\eta_{1}}(f \circ \psi)\left(\psi^{-1}\left(v_{2}\right)\right)+\frac{1-\eta_{1}}{B\left(\eta_{1}\right)}(f \circ \psi)\left(\psi^{-1}\left(v_{2}\right)\right)\right] \\
& +\left[\frac{\eta_{1}}{B\left(\eta_{1}\right)}{ }^{R L}(x) \mathrm{J}_{\psi^{-1}\left(v_{2}\right)-}^{\eta_{1}}(f \circ \psi)\left(\psi^{-1}\left(v_{1}\right)\right)+\frac{1-\eta_{1}}{B\left(\eta_{1}\right)}(f \circ \psi)\left(\psi^{-1}\left(v_{1}\right)\right)\right] \\
& =\frac{\eta_{1}}{B\left(\eta_{1}\right)}\left[\underset{\psi(x)}{R L} \mathrm{~J}_{\psi^{-1}\left(v_{1}\right)+}^{\eta_{1}}(f \circ \psi)\left(\psi^{-1}\left(v_{2}\right)\right)+\underset{\psi(x)}{R L} \mathrm{~J}_{\psi^{-1}\left(v_{2}\right)-}^{\eta_{1}}(f \circ \psi)\left(\psi^{-1}\left(v_{1}\right)\right)\right] \\
& +\frac{1-\eta_{1}}{B\left(\eta_{1}\right)}\left[f\left(v_{1}\right)+f\left(v_{2}\right)\right]
\end{aligned}
$$

Since the quantities $\Gamma\left(\eta_{1}+1\right)$ and $2\left(v_{2}-v_{1}\right)^{\eta_{1}}$ are positive, then by using inequality (18), we get

$$
\begin{aligned}
& \frac{2\left(v_{2}-v_{1}\right)^{\eta_{1}}}{\Gamma\left(\eta_{1}+1\right)} f\left(\frac{v_{1}+v_{2}}{2}\right) \\
& \quad \leq \underset{\psi(x)}{R L} J_{\psi^{-1}\left(v_{1}\right)+}^{\eta_{1}}(f \circ \psi)\left(\psi^{-1}\left(v_{2}\right)\right)+{ }_{\psi(x)}^{R L} J_{\psi^{-1}\left(v_{2}\right)-}^{\eta_{1}}(f \circ \psi)\left(\psi^{-1}\left(v_{1}\right)\right) \\
& \quad \leq \frac{2\left(v_{2}-v_{1}\right)^{\eta_{1}}}{\Gamma\left(\eta_{1}+1\right)} \cdot \frac{f\left(v_{1}\right)+f\left(v_{2}\right)}{2} .
\end{aligned}
$$

By multiplying by $\frac{\eta_{1}}{B\left(\eta_{1}\right)}$ and adding $\frac{1-\eta_{1}}{B\left(\eta_{1}\right)}\left[f\left(v_{1}\right)+f\left(v_{2}\right)\right]$, we get AB integrals in the middle:

$$
\begin{aligned}
& \frac{2\left(v_{2}-v_{1}\right)^{\eta_{1}}}{\Gamma\left(\eta_{1}\right) B\left(\eta_{1}\right)} f\left(\frac{v_{1}+v_{2}}{2}\right)+\frac{1-\eta_{1}}{B\left(\eta_{1}\right)}\left[f\left(v_{1}\right)+f\left(v_{2}\right)\right] \\
& \quad \leq \underset{\psi(x) J_{\psi^{-1}\left(v_{1}\right)+}^{\eta_{1}}}{ }(f \circ \psi)\left(\psi^{-1}\left(v_{2}\right)\right)+\underset{\psi(x)}{A B} \mathrm{~J}_{\psi^{-1}\left(v_{2}\right)-}^{\eta_{1}}(f \circ \psi)\left(\psi^{-1}\left(v_{1}\right)\right) \\
& \quad \leq \frac{2\left(v_{2}-v_{1}\right)^{\eta_{1}}}{\Gamma\left(\eta_{1}\right) B\left(\eta_{1}\right)} \cdot \frac{f\left(v_{1}\right)+f\left(v_{2}\right)}{2}+\frac{1-\eta_{1}}{B\left(\eta_{1}\right)}\left[f\left(v_{1}\right)+f\left(v_{2}\right)\right] .
\end{aligned}
$$


By convexity of $f$, we have $f\left(\frac{v_{1}+v_{2}}{2}\right) \leq \frac{f\left(v_{1}\right)+f\left(v_{2}\right)}{2}$, so we can deduce

$$
\begin{aligned}
& {\left[\frac{2\left(v_{2}-v_{1}\right)^{\eta_{1}}}{\Gamma\left(\eta_{1}\right) B\left(\eta_{1}\right)}+\frac{2\left(1-\eta_{1}\right)}{B\left(\eta_{1}\right)}\right] f\left(\frac{v_{1}+v_{2}}{2}\right)} \\
& \quad \leq \underset{\psi(x)}{A B} \mathrm{~J}_{\psi^{-1}\left(v_{1}\right)+}^{\eta_{1}}(f \circ \psi)\left(\psi^{-1}\left(v_{2}\right)\right)+\underset{\psi(x)}{A B} \mathrm{~J}_{\psi^{-1}\left(v_{2}\right)-}^{\eta_{1}}(f \circ \psi)\left(\psi^{-1}\left(v_{1}\right)\right) \\
& \quad \leq\left[\frac{2\left(v_{2}-v_{1}\right)^{\eta_{1}}}{\Gamma\left(\eta_{1}\right) B\left(\eta_{1}\right)}+\frac{2\left(1-\eta_{1}\right)}{B\left(\eta_{1}\right)}\right] \frac{f\left(v_{1}\right)+f\left(v_{2}\right)}{2},
\end{aligned}
$$

which rearranges to the required result.

Corollary 3.1 Theorem 3.1 with $\psi(x)=x$ becomes

$$
\begin{aligned}
f\left(\frac{\nu_{1}+v_{2}}{2}\right) & \leq \frac{B\left(\eta_{1}\right) \Gamma\left(\eta_{1}\right)}{2\left[\left(v_{2}-v_{1}\right)^{\eta_{1}}+\left(1-\eta_{1}\right) \Gamma\left(\eta_{1}\right)\right]}\left[{ }^{A B} \mathcal{J}_{v_{1} f}^{\eta_{1}} f\left(v_{2}\right)+{ }^{A B} \mathrm{~J}_{v_{2}}^{\eta_{1}} f\left(v_{1}\right)\right] \\
& \leq \frac{f\left(v_{1}\right)+f\left(v_{2}\right)}{2}
\end{aligned}
$$

which is proved by Fernandez and Mohammed in [19, Proposition 2.1].

Theorem 3.2 Iff $:\left[v_{1}, v_{2}\right] \rightarrow \mathfrak{R}$ is an $\mathrm{L}^{1}$ convex function, $\psi$ is an increasing positive function on $\left(v_{1}, v_{2}\right]$ with $\psi^{\prime}(x) \in \mathrm{L}^{1}\left(v_{1}, v_{2}\right)$, and $\eta_{1} \in(0,1)$, then we have

$$
\begin{aligned}
& \mathcal{Q}_{1}\left(\eta_{1}, v_{2}-v_{1}\right) f\left(\frac{v_{1}+v_{2}}{2}\right)+\mathcal{Q}_{2}\left(\eta_{1}, v_{2}-v_{1}\right) \frac{f\left(v_{1}\right)+f\left(v_{2}\right)}{2} \\
& \leq \frac{1-\eta_{1}}{2 B\left(\eta_{1}\right) E_{\eta_{1}}\left(\frac{-\eta_{1}}{1-\eta_{1}}\left(v_{2}-v_{1}\right)^{\eta_{1}}\right)} \\
& \times\left[{ }_{\psi(x)}^{A B R} \mathrm{D}_{\psi^{-1}\left(v_{1}\right)+}^{\eta_{1}}(f \circ \psi)\left(\psi^{-1}\left(v_{2}\right)\right)+{ }_{\psi(x)}^{A B R} \mathrm{D}_{\psi^{-1}\left(v_{2}\right)_{-}}^{\eta_{1}}(f \circ \psi)\left(\psi^{-1}\left(v_{1}\right)\right)\right] \\
& \leq \mathcal{Q}_{2}\left(\eta_{1}, v_{2}-v_{1}\right) f\left(\frac{v_{1}+v_{2}}{2}\right)+\mathcal{Q}_{1}\left(\eta_{1}, v_{2}-v_{1}\right) \frac{f\left(v_{1}\right)+f\left(v_{2}\right)}{2} \text {, }
\end{aligned}
$$

where the multipliers $\mathcal{Q}_{1}\left(\eta_{1}, v_{2}-v_{1}\right)$ and $\mathcal{Q}_{2}\left(\eta_{1}, v_{2}-v_{1}\right)$ sum to 1 , therefore organizing weighted averages on both ends of the inequality, and these are defined as follows:

$$
\begin{aligned}
& \mathcal{Q}_{1}\left(\eta_{1}, \nu_{2}-v_{1}\right)=\frac{E_{2 \eta_{1}}\left(\left(\frac{-\eta_{1}}{1-\eta_{1}}\right)^{2}\left(\nu_{2}-v_{1}\right)^{2 \eta_{1}}\right)}{E_{\eta_{1}}\left(\frac{-\eta_{1}}{1-\eta_{1}}\left(\nu_{2}-v_{1}\right)^{\eta_{1}}\right)} ; \\
& \mathcal{Q}_{2}\left(\eta_{1}, \nu_{2}-v_{1}\right)=\frac{\frac{-\eta_{1}}{1-\eta_{1}}\left(\nu_{2}-v_{1}\right)^{\eta_{1}} E_{2 \eta_{1}, \eta_{1}+1}\left(\left(\frac{-\eta_{1}}{1-\eta_{1}}\right)^{2}\left(v_{2}-v_{1}\right)^{2 \eta_{1}}\right)}{E_{\eta_{1}}\left(\frac{-\eta_{1}}{1-\eta_{1}}\left(v_{2}-v_{1}\right)^{\eta_{1}}\right)} .
\end{aligned}
$$

Proof Following [17], we have the following key result:

$$
\underset{\psi(x)}{A B R} \mathrm{D}_{\psi^{-1}\left(\nu_{1}\right)}^{\eta_{1}} f(x)=\frac{B\left(\eta_{1}\right)}{1-\eta_{1}} \sum_{k=0}^{\infty}\left(\frac{-\eta_{1}}{1-\eta_{1}}\right)^{k} \underset{\psi(x)}{R L} \mathrm{~J}_{\psi^{-1}\left(\nu_{1}\right)+}^{k \eta_{1}} f(x) .
$$

The series being locally uniformly convergent (see [17]). From this formula, we see that the $A B$ derivatives can be written purely in terms of RL integrals. In view of this result, many results can be proved in the $\mathrm{AB}$ model directly from the corresponding results in the RL model. 
Here, we apply the $\mathrm{HH}$-inequality for fractional $\psi$-RL integrals (18) with $\eta_{1}$ replaced by $k \eta_{1}$ :

$$
\begin{aligned}
f\left(\frac{v_{1}+v_{2}}{2}\right) \leq & \frac{\Gamma\left(k \eta_{1}+1\right)}{2\left(v_{2}-v_{1}\right)^{k \eta_{1}}}\left[\begin{array}{c}
R L \\
\psi(x) \\
\mathrm{J}_{\psi^{-1}\left(v_{1}\right)_{+}}^{k \eta_{1}}
\end{array}\right. \\
& \left.+\underset{\psi(x)}{R L} \mathrm{~J}_{\psi^{-1}\left(v_{2}\right)_{-}}^{k \eta_{1}}(f \circ \psi)\left(\psi^{-1}\left(v_{1}\right)\right)\right] \\
\leq & \frac{f\left(v_{1}\right)+f\left(v_{2}\right)}{2} .
\end{aligned}
$$

Multiplying on all sides of the inequality by positive constants, we get

$$
\begin{aligned}
& \frac{2\left(v_{2}-v_{1}\right)^{k \eta_{1}}}{\Gamma\left(k \eta_{1}+1\right)} f\left(\frac{v_{1}+v_{2}}{2}\right) \\
& \quad \leq \begin{array}{c}
R L \\
\psi(x) \\
\mathrm{J}_{\psi}^{-1}\left(v_{1}\right)+
\end{array}(f \circ \psi)\left(\psi^{-1}\left(v_{2}\right)\right)+\underset{\psi(x)}{R L} \mathrm{~J}_{\psi^{-1}\left(v_{2}\right)-}^{k \eta_{1}}(f \circ \psi)\left(\psi^{-1}\left(v_{1}\right)\right) \\
& \quad \leq \frac{2\left(v_{2}-v_{1}\right)^{k \eta_{1}}}{\Gamma\left(k \eta_{1}+1\right)} \cdot \frac{f\left(v_{1}\right)+f\left(v_{2}\right)}{2} .
\end{aligned}
$$

The constant $\frac{-\eta_{1}}{1-\eta_{1}}$ is negative since $0<\eta_{1}<1$. So, we need to split the above inequality into two cases according to the parity of $k$.

If $k$ is even, then we have

$$
\begin{aligned}
& \left(\frac{-\eta_{1}}{1-\eta_{1}}\right)^{k} \frac{2\left(v_{2}-v_{1}\right)^{k \eta_{1}}}{\Gamma\left(k \eta_{1}+1\right)} f\left(\frac{v_{1}+v_{2}}{2}\right) \\
& \quad \leq\left(\frac{-\eta_{1}}{1-\eta_{1}}\right)^{k}\left[\begin{array}{c}
R L \\
\psi(x)
\end{array} \mathrm{J}_{\psi^{-1}\left(v_{1}\right)+}^{k \eta_{1}}(f \circ \psi)\left(\psi^{-1}\left(v_{2}\right)\right)+\underset{\psi(x)}{R L} \mathrm{~J}_{\psi^{-1}\left(v_{2}\right)-}^{k \eta_{1}}(f \circ \psi)\left(\psi^{-1}\left(v_{1}\right)\right)\right] \\
& \quad \leq\left(\frac{-\eta_{1}}{1-\eta_{1}}\right)^{k} \frac{2\left(v_{2}-v_{1}\right)^{k \eta_{1}}}{\Gamma\left(k \eta_{1}+1\right)} \cdot \frac{f\left(v_{1}\right)+f\left(v_{2}\right)}{2} .
\end{aligned}
$$

If $k$ is odd, then we have

$$
\begin{aligned}
& \left(\frac{-\eta_{1}}{1-\eta_{1}}\right)^{k} \frac{2\left(v_{2}-v_{1}\right)^{k \eta_{1}}}{\Gamma\left(k \eta_{1}+1\right)} \cdot \frac{f\left(v_{1}\right)+f\left(v_{2}\right)}{2}
\end{aligned}
$$

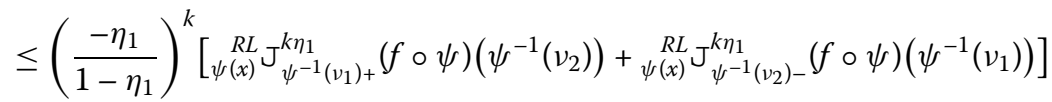

$$
\begin{aligned}
& \leq\left(\frac{-\eta_{1}}{1-\eta_{1}}\right)^{k} \frac{2\left(v_{2}-v_{1}\right)^{k \eta_{1}}}{\Gamma\left(k \eta_{1}+1\right)} f\left(\frac{v_{1}+v_{2}}{2}\right) \text {. }
\end{aligned}
$$

Summing over all $k$ and making use of the series formula (22), we deduce

$$
\begin{aligned}
& \sum_{k \text { even }}\left(\frac{-\eta_{1}}{1-\eta_{1}}\right)^{k} \frac{2\left(v_{2}-v_{1}\right)^{k \eta_{1}}}{\Gamma\left(k \eta_{1}+1\right)} f\left(\frac{v_{1}+v_{2}}{2}\right) \\
& \quad+\sum_{k \text { odd }}\left(\frac{-\eta_{1}}{1-\eta_{1}}\right)^{k} \frac{2\left(v_{2}-v_{1}\right)^{k \eta_{1}}}{\Gamma\left(k \eta_{1}+1\right)} \cdot \frac{f\left(v_{1}\right)+f\left(v_{2}\right)}{2} \\
& \leq \frac{1-\eta_{1}}{B\left(\eta_{1}\right)}\left[\begin{array}{l}
A B R \\
\psi(x)
\end{array} \mathrm{J}_{\psi^{-1}\left(v_{1}\right)+}^{\eta_{1}}(f \circ \psi)\left(\psi^{-1}\left(v_{2}\right)\right)+{ }_{\psi(x)}^{A B R} \mathrm{~J}_{\psi^{-1}\left(v_{2}\right)-}^{\eta_{1}}(f \circ \psi)\left(\psi^{-1}\left(v_{1}\right)\right)\right]
\end{aligned}
$$




$$
\begin{aligned}
\leq & \sum_{k \text { even }}\left(\frac{-\eta_{1}}{1-\eta_{1}}\right)^{k} \frac{2\left(v_{2}-v_{1}\right)^{k \eta_{1}}}{\Gamma\left(k \eta_{1}+1\right)} \cdot \frac{f\left(v_{1}\right)+f\left(v_{2}\right)}{2} \\
& +\sum_{k \text { odd }}\left(\frac{-\eta_{1}}{1-\eta_{1}}\right)^{k} \frac{2\left(v_{2}-v_{1}\right)^{k \eta_{1}}}{\Gamma\left(k \eta_{1}+1\right)} f\left(\frac{v_{1}+v_{2}}{2}\right) .
\end{aligned}
$$

The sums over even and odd $k$ can be rewritten more precisely as follows:

$$
\begin{aligned}
& \sum_{n=0}^{\infty}\left(\frac{-\eta_{1}}{1-\eta_{1}}\right)^{2 n} \frac{2\left(v_{2}-v_{1}\right)^{2 n \eta_{1}}}{\Gamma\left(2 n \eta_{1}+1\right)} f\left(\frac{v_{1}+v_{2}}{2}\right) \\
& +\sum_{n=0}^{\infty}\left(\frac{-\eta_{1}}{1-\eta_{1}}\right)^{2 n+1} \frac{2\left(v_{2}-v_{1}\right)^{(2 n+1) \eta_{1}}}{\Gamma\left((2 n+1) \eta_{1}+1\right)} \cdot \frac{f\left(v_{1}\right)+f\left(v_{2}\right)}{2}
\end{aligned}
$$

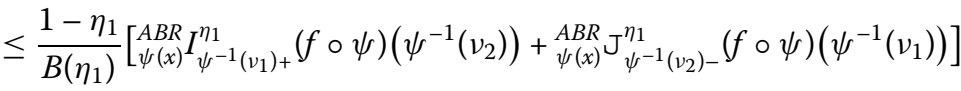

$$
\begin{aligned}
& \leq \sum_{n=0}^{\infty}\left(\frac{-\eta_{1}}{1-\eta_{1}}\right)^{2 n} \frac{2\left(v_{2}-v_{1}\right)^{2 n \eta_{1}}}{\Gamma\left(2 n \eta_{1}+1\right)} \cdot \frac{f\left(v_{1}\right)+f\left(v_{2}\right)}{2} \\
& +\sum_{n=0}^{\infty}\left(\frac{-\eta_{1}}{1-\eta_{1}}\right)^{2 n+1} \frac{2\left(v_{2}-v_{1}\right)^{(2 n+1) \eta_{1}}}{\Gamma\left((2 n+1) \eta_{1}+1\right)} f\left(\frac{\nu_{1}+v_{2}}{2}\right) .
\end{aligned}
$$

The infinite series appearing here can be re-explained as the power series for ML functions:

$$
\begin{aligned}
& 2 E_{2 \eta_{1}}\left(\left(\frac{-\eta_{1}}{1-\eta_{1}}\right)^{2}\left(v_{2}-v_{1}\right)^{2 \eta_{1}}\right) f\left(\frac{\nu_{1}+v_{2}}{2}\right) \\
& +2\left(\frac{-\eta_{1}}{1-\eta_{1}}\right)\left(v_{2}-v_{1}\right)^{\eta_{1}} E_{2 \eta_{1}, \eta_{1}+1}\left(\left(\frac{-\eta_{1}}{1-\eta_{1}}\right)^{2}\left(v_{2}-v_{1}\right)^{2 \eta_{1}}\right) \frac{f\left(v_{1}\right)+f\left(v_{2}\right)}{2} \\
& \leq \frac{1-\eta_{1}}{B\left(\eta_{1}\right)}\left[\begin{array}{l}
A B R \\
\psi(x)
\end{array} \mathrm{J}_{\psi^{-1}\left(v_{1}\right)+}^{\eta_{1}}(f \circ \psi)\left(\psi^{-1}\left(v_{2}\right)\right)+{ }_{\psi(x)}^{A B R} \mathrm{~J}_{\psi^{-1}\left(v_{2}\right)-}^{\eta_{1}}(f \circ \psi)\left(\psi^{-1}\left(v_{1}\right)\right)\right] \\
& \leq 2 E_{2 \eta_{1}}\left(\left(\frac{-\eta_{1}}{1-\eta_{1}}\right)^{2}\left(v_{2}-v_{1}\right)^{2 \eta_{1}}\right) \frac{f\left(v_{1}\right)+f\left(v_{2}\right)}{2} \\
& +2\left(\frac{-\eta_{1}}{1-\eta_{1}}\right)\left(v_{2}-v_{1}\right)^{\eta_{1}} E_{2 \eta_{1}, \eta_{1}+1}\left(\left(\frac{-\eta_{1}}{1-\eta_{1}}\right)^{2}\left(v_{2}-v_{1}\right)^{2 \eta_{1}}\right) f\left(\frac{\nu_{1}+v_{2}}{2}\right) \text {. }
\end{aligned}
$$

We observe that the two functions $E_{2 \eta_{1}}\left(\left(\frac{-\eta_{1}}{1-\eta_{1}}\right)^{2}\left(\nu_{2}-v_{1}\right)^{2 \eta_{1}}\right)$ and $\left(\frac{-\eta_{1}}{1-\eta_{1}}\right)\left(v_{2}-v_{1}\right)^{\eta_{1}} \times$ $E_{2 \eta_{1}, \eta_{1}+1}\left(\left(\frac{-\eta_{1}}{1-\eta_{1}}\right)^{2}\left(v_{2}-v_{1}\right)^{2 \eta_{1}}\right)$ that appear as multipliers, since they arise from the even and odd parts of a single infinite series, become a simpler ML function when they are added together:

$$
\begin{aligned}
& E_{2 \eta_{1}}\left(\left(\frac{-\eta_{1}}{1-\eta_{1}}\right)^{2}\left(v_{2}-v_{1}\right)^{2 \eta_{1}}\right)+\left(\frac{-\eta_{1}}{1-\eta_{1}}\right)\left(v_{2}-v_{1}\right)^{\eta_{1}} E_{2 \eta_{1}, \eta_{1}+1}\left(\left(\frac{-\eta_{1}}{1-\eta_{1}}\right)^{2}\left(v_{2}-v_{1}\right)^{2 \eta_{1}}\right) \\
& =\sum_{k \text { even }}\left(\frac{-\eta_{1}}{1-\eta_{1}}\right)^{k} \frac{\left(v_{2}-v_{1}\right)^{k \eta_{1}}}{\Gamma\left(k \eta_{1}+1\right)}+\sum_{k \text { odd }}\left(\frac{-\eta_{1}}{1-\eta_{1}}\right)^{k} \frac{\left(v_{2}-v_{1}\right)^{k \eta_{1}}}{\Gamma\left(k \eta_{1}+1\right)}
\end{aligned}
$$




$$
\begin{aligned}
& =\sum_{k=0}^{\infty}\left(\frac{-\eta_{1}}{1-\eta_{1}}\right)^{k} \frac{\left(v_{2}-v_{1}\right)^{k \eta_{1}}}{\Gamma\left(k \eta_{1}+1\right)} \\
& =E_{\eta_{1}}\left(\frac{-\eta_{1}}{1-\eta_{1}}\left(v_{2}-v_{1}\right)^{\eta_{1}}\right) .
\end{aligned}
$$

Also, the sum function $E_{\eta_{1}}\left(\frac{-\eta_{1}}{1-\eta_{1}}\left(v_{2}-v_{1}\right)^{\eta_{1}}\right)$ is strictly positive [45], so it allows us to divide by it in inequality (23) to get

$$
\begin{aligned}
& \frac{E_{2 \eta_{1}}\left(\left(\frac{-\eta_{1}}{1-\eta_{1}}\right)^{2}\left(v_{2}-v_{1}\right)^{2 \eta_{1}}\right)}{E_{\eta_{1}}\left(\frac{-\eta_{1}}{1-\eta_{1}}\left(v_{2}-v_{1}\right)^{\eta_{1}}\right)} f\left(\frac{\nu_{1}+v_{2}}{2}\right) \\
& +\frac{\frac{-\eta_{1}}{1-\eta_{1}}\left(\nu_{2}-v_{1}\right)^{\eta_{1}} E_{2 \eta_{1}, \eta_{1}+1}\left(\left(\frac{-\eta_{1}}{1-\eta_{1}}\right)^{2}\left(v_{2}-v_{1}\right)^{2 \eta_{1}}\right)}{E_{\eta_{1}}\left(\frac{-\eta_{1}}{1-\eta_{1}}\left(v_{2}-v_{1}\right)^{\eta_{1}}\right)} \cdot \frac{f\left(v_{1}\right)+f\left(v_{2}\right)}{2} \\
& \leq \frac{1-\eta_{1}}{2 B\left(\eta_{1}\right) E_{\eta_{1}}\left(\frac{-\eta_{1}}{1-\eta_{1}}\left(v_{2}-v_{1}\right)^{\eta_{1}}\right)} \\
& \times\left[{ }_{\psi(x)}^{A B R} \mathrm{~J}_{\psi^{-1}\left(v_{1}\right)_{+}}^{\eta_{1}}(f \circ \psi)\left(\psi^{-1}\left(v_{2}\right)\right)+{ }_{\psi(x)}^{A B R} \mathrm{~J}_{\psi^{-1}\left(v_{2}\right)_{-}}^{\eta_{1}}(f \circ \psi)\left(\psi^{-1}\left(v_{1}\right)\right)\right] \\
& \leq \frac{E_{2 \eta_{1}}\left(\left(\frac{-\eta_{1}}{1-\eta_{1}}\right)^{2}\left(v_{2}-v_{1}\right)^{2 \eta_{1}}\right)}{E_{\eta_{1}}\left(\frac{-\eta_{1}}{1-\eta_{1}}\left(v_{2}-v_{1}\right)^{\eta_{1}}\right)} \cdot \frac{f\left(v_{1}\right)+f\left(v_{2}\right)}{2} \\
& +\frac{\frac{-\eta_{1}}{1-\eta_{1}}\left(v_{2}-v_{1}\right)^{\eta_{1}} E_{2 \eta_{1}, \eta_{1}+1}\left(\left(\frac{-\eta_{1}}{1-\eta_{1}}\right)^{2}\left(v_{2}-v_{1}\right)^{2 \eta_{1}}\right)}{E_{\eta_{1}}\left(\frac{-\eta_{1}}{1-\eta_{1}}\left(v_{2}-v_{1}\right)^{\eta_{1}}\right)} f\left(\frac{\nu_{1}+v_{2}}{2}\right),
\end{aligned}
$$

which is the required result.

Corollary 3.2 Theorem 3.2 with $\psi(x)=x$ becomes

$$
\begin{aligned}
& \mathcal{Q}_{1}\left(\eta_{1}, v_{2}-v_{1}\right) f\left(\frac{v_{1}+v_{2}}{2}\right)+\mathcal{Q}_{2}\left(\eta_{1}, v_{2}-v_{1}\right) \frac{f\left(v_{1}\right)+f\left(v_{2}\right)}{2} \\
& \leq \frac{1-\eta_{1}}{2 B\left(\eta_{1}\right) E_{\eta_{1}}\left(\frac{-\eta_{1}}{1-\eta_{1}}\left(v_{2}-v_{1}\right)\right.}\left[{ }^{\left.\eta_{1}\right)}\left[{ }^{A B R} \mathrm{D}_{v_{1}+}^{\eta_{1}} f\left(v_{2}\right)+{ }^{A B R} \mathrm{D}_{v_{2}}^{\eta_{1}} f\left(v_{1}\right)\right]\right. \\
& \leq \mathcal{Q}_{2}\left(\eta_{1}, v_{2}-v_{1}\right) f\left(\frac{v_{1}+v_{2}}{2}\right)+\mathcal{Q}_{1}\left(\eta_{1}, v_{2}-v_{1}\right) \frac{f\left(v_{1}\right)+f\left(v_{2}\right)}{2},
\end{aligned}
$$

where the multipliers $\mathcal{Q}_{1}\left(\eta_{1}, v_{2}-v_{1}\right)$ and $\mathcal{Q}_{2}\left(\eta_{1}, v_{2}-v_{1}\right)$ are defined as follows:

$$
\begin{aligned}
& \mathcal{Q}_{1}\left(\eta_{1}, v_{2}-v_{1}\right)=\frac{E_{2 \eta_{1}}\left(\left(\frac{-\eta_{1}}{1-\eta_{1}}\right)^{2}\left(v_{2}-v_{1}\right)^{2 \eta_{1}}\right)}{E_{\eta_{1}}\left(\frac{-\eta_{1}}{1-\eta_{1}}\left(v_{2}-v_{1}\right)^{\eta_{1}}\right)} ; \\
& \mathcal{Q}_{2}\left(\eta_{1}, v_{2}-v_{1}\right)=\frac{\frac{-\eta_{1}}{1-\eta_{1}}\left(v_{2}-v_{1}\right)^{\eta_{1}} E_{2 \eta_{1}, \eta_{1}+1}\left(\left(\frac{-\eta_{1}}{1-\eta_{1}}\right)^{2}\left(v_{2}-v_{1}\right)^{2 \eta_{1}}\right)}{E_{\eta_{1}}\left(\frac{-\eta_{1}}{1-\eta_{1}}\left(v_{2}-v_{1}\right)^{\eta_{1}}\right)},
\end{aligned}
$$

which is proved by Fernandez and Mohammed in [19, Theorem 2.1].

Example 3.1 We verify the result of Theorem 3.2 for $\psi(x)=x$ and the convex function $f(x)=e^{3 x}$. Due to [32], we have

$$
{ }^{R L} \mathrm{~J}_{c^{+}} e^{\eta_{1}} e^{3 x}=e^{3 x} 3^{-\eta_{1}} \frac{\gamma\left(\eta_{1}, 3(x-c)\right)}{\Gamma\left(\eta_{1}\right)}, \quad R{ }_{\mathrm{J}_{c-}}^{\eta_{1}} e^{3 x}=e^{3 x}(-3)^{-\eta_{1}} \frac{\gamma\left(\eta_{1}, 3(x-c)\right)}{\Gamma\left(\eta_{1}\right)} .
$$


By making use of the series formula (22), we have

$$
\begin{aligned}
{ }^{A B R} \mathrm{D}_{c+}^{\eta_{1}} e^{3 x} & =\frac{B\left(\eta_{1}\right)}{1-\eta_{1}} e^{3 x} \sum_{k=0}^{\infty}\left(\frac{-\eta_{1}}{1-\eta_{1}}\right)^{k} 3^{-k \eta_{1}} \frac{\gamma\left(k \eta_{1}, 3(x-c)\right)}{\Gamma\left(k \eta_{1}\right)}, \\
{ }^{A B R} \mathrm{D}_{c-}^{\eta_{1}} e^{3 x} & =\frac{B\left(\eta_{1}\right)}{1-\eta_{1}} e^{3 x} \sum_{k=0}^{\infty}\left(\frac{-\eta_{1}}{1-\eta_{1}}\right)^{k}(-3)^{-k \eta_{1}} \frac{\gamma\left(k \eta_{1}, 3(x-c)\right)}{\Gamma\left(k \eta_{1}\right)} .
\end{aligned}
$$

Substituting the above expressions into $\mathrm{HH}$-inequality (21), we get

$$
\begin{aligned}
\mathcal{Q}_{1}\left(\eta_{1}, v_{2}-v_{1}\right) e^{3\left(v_{1}+v_{2}\right) / 2}+\mathcal{Q}_{2}\left(\eta_{1}, v_{2}-v_{1}\right) \frac{e^{3 \nu_{1}}+e^{3 v_{2}}}{2} \\
\leq \frac{1-\eta_{1}}{2 B\left(\eta_{1}\right) E_{\eta_{1}}\left(\frac{-\eta_{1}}{1-\eta_{1}}\left(v_{2}-v_{1}\right)^{\eta_{1}}\right)}\left[\frac{B\left(\eta_{1}\right)}{1-\eta_{1}} e^{3 v_{2}} \sum_{k=0}^{\infty}\left(\frac{-\eta_{1}}{1-\eta_{1}}\right)^{k} 3^{-k \eta_{1}} \frac{\gamma\left(k \eta_{1}, 3\left(v_{2}-v_{1}\right)\right)}{\Gamma\left(k \eta_{1}\right)}\right. \\
\left.\quad+\frac{B\left(\eta_{1}\right)}{1-\eta_{1}} e^{3 v_{1}} \sum_{k=0}^{\infty}\left(\frac{-\eta_{1}}{1-\eta_{1}}\right)^{k}(-3)^{-k \eta_{1}} \frac{\gamma\left(k \eta_{1}, 3\left(v_{1}-b\right)\right)}{\Gamma\left(k \eta_{1}\right)}\right] \\
\leq \mathcal{Q}_{2}\left(\eta_{1}, v_{2}-v_{1}\right) e^{3\left(v_{1}+v_{2}\right) / 2}+\mathcal{Q}_{1}\left(\eta_{1}, v_{2}-v_{1}\right) \frac{e^{3 v_{1}}+e^{3 v_{2}}}{2} .
\end{aligned}
$$

Additional simplification process gives

$$
\begin{aligned}
\mathcal{Q}_{1}\left(\eta_{1}, v_{2}-v_{1}\right) e^{3\left(v_{1}+v_{2}\right) / 2}+\mathcal{Q}_{2}\left(\eta_{1}, v_{2}-v_{1}\right) \frac{e^{3 \nu_{1}}+e^{3 \nu_{2}}}{2} \\
\leq \frac{1}{2 E_{\eta_{1}}\left(\frac{-\eta_{1}}{1-\eta_{1}}\left(v_{2}-v_{1}\right)^{\left.\eta_{1}\right)}\right.} \\
\quad \times \sum_{k=0}^{\infty} \frac{\left(\frac{-\eta_{1}}{1-\eta_{1}}\right)^{k}}{\Gamma\left(k \eta_{1}\right)}\left[3^{-k \eta_{1}} e^{3 v_{2}} \gamma\left(k \eta_{1}, 3\left(v_{2}-v_{1}\right)\right)+(-3)^{-k \eta_{1}} e^{3 v_{1}} \gamma\left(k \eta_{1}, 3\left(v_{1}-b\right)\right)\right] \\
\leq \mathcal{Q}_{2}\left(\eta_{1}, v_{2}-v_{1}\right) e^{3\left(v_{1}+v_{2}\right) / 2}+\mathcal{Q}_{1}\left(\eta_{1}, v_{2}-v_{1}\right) \frac{e^{3 v_{1}}+e^{3 v_{2}}}{2}
\end{aligned}
$$

or dropping the multiplier $\mathcal{Q}$ leads to

$$
\begin{aligned}
& 2 E_{2 \eta_{1}}\left(\left(\frac{-\eta_{1}}{1-\eta_{1}}\right)^{2}\left(v_{2}-v_{1}\right)^{2 \eta_{1}}\right) e^{3\left(v_{1}+v_{2}\right) / 2} \\
& \quad+\frac{-\eta_{1}}{1-\eta_{1}}\left(v_{2}-v_{1}\right)^{\eta_{1}} E_{2 \eta_{1}, \eta_{1}+1}\left(\left(\frac{-\eta_{1}}{1-\eta_{1}}\right)^{2}\left(v_{2}-v_{1}\right)^{2 \eta_{1}}\right)\left(e^{3 v_{1}}+e^{3 v_{2}}\right) \\
& \leq \sum_{k=0}^{\infty} \frac{\left(\frac{-\eta_{1}}{1-\eta_{1}}\right)^{k}}{\Gamma\left(k \eta_{1}\right)}\left[3^{-k \eta_{1}} e^{3 v_{2}} \gamma\left(k \eta_{1}, 3\left(v_{2}-v_{1}\right)\right)+(-3)^{-k \eta_{1}} e^{3 v_{1}} \gamma\left(k \eta_{1}, 3\left(v_{1}-b\right)\right)\right] \\
& \leq 2 \frac{-\eta_{1}}{1-\eta_{1}}\left(v_{2}-v_{1}\right)^{\eta_{1}} E_{2 \eta_{1}, \eta_{1}+1}\left(\left(\frac{-\eta_{1}}{1-\eta_{1}}\right)^{2}\left(v_{2}-v_{1}\right)^{2 \eta_{1}}\right) e^{3\left(v_{1}+v_{2}\right) / 2} \\
& \quad+E_{2 \eta_{1}}\left(\left(\frac{-\eta_{1}}{1-\eta_{1}}\right)^{2}\left(v_{2}-v_{1}\right)^{2 \eta_{1}}\right)\left(e^{3 v_{1}}+e^{3 v_{2}}\right) .
\end{aligned}
$$


Figure 1 A plot illustration for Example 3.1

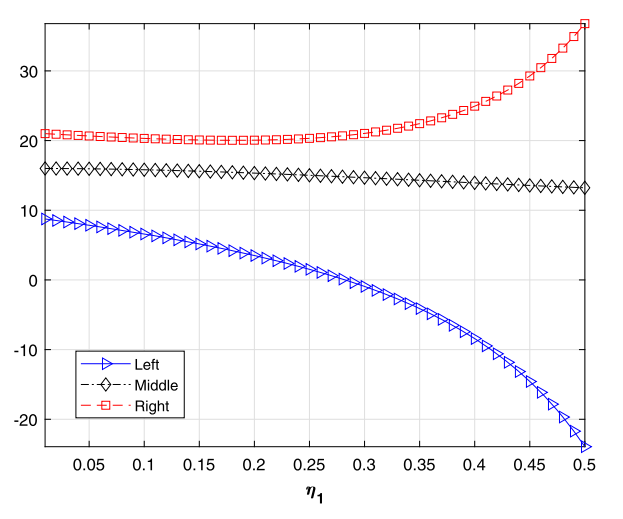

For a specific value of $\eta_{1} \in\left(0, \frac{1}{2}\right)$ and $v_{1}=0, v_{2}=1$, this inequality becomes

$$
\begin{aligned}
& 2 E_{2 \eta_{1}}\left(\left(\frac{-\eta_{1}}{1-\eta_{1}}\right)^{2}\right) e^{3 / 2}+\frac{-\eta_{1}}{1-\eta_{1}} E_{2 \eta_{1}, \eta_{1}+1}\left(\left(\frac{-\eta_{1}}{1-\eta_{1}}\right)^{2}\right)\left(1+e^{3}\right) \\
& \quad \leq \sum_{k=0}^{\infty} \frac{\left(\frac{-\eta_{1}}{1-\eta_{1}}\right)^{k}}{\Gamma\left(k \eta_{1}\right)}\left[3^{-k \eta_{1}} e^{3} \gamma\left(k \eta_{1}, 3\right)+(-3)^{-k \eta_{1}} \gamma\left(k \eta_{1},-3\right)\right] \\
& \quad \leq 2 \frac{-\eta_{1}}{1-\eta_{1}} E_{2 \eta_{1}, \eta_{1}+1}\left(\left(\frac{-\eta_{1}}{1-\eta_{1}}\right)^{2}\right) e^{3 / 2}+E_{2 \eta_{1}}\left(\left(\frac{-\eta_{1}}{1-\eta_{1}}\right)^{2}\right)\left(1+e^{3}\right) .
\end{aligned}
$$

The three functions given by the left-, middle-, and right-hand sides of the double inequality (25) are plotted in Fig. 1 against $\eta_{1} \in\left(0, \frac{1}{2}\right)$ to demonstrate clearly that both inequalities are valid.

Theorem 3.3 Iff $:\left[v_{1}, v_{2}\right] \rightarrow \Re$ is an $\mathrm{L}^{1}$ convex function, $\psi$ is an increasing positive function on $\left(v_{1}, v_{2}\right]$ with $\psi^{\prime}(x) \in \mathrm{L}^{1}\left(v_{1}, v_{2}\right)$, and $\eta_{1}, \eta_{2}, \gamma, \omega>0$, then we have

$$
\begin{aligned}
f\left(\frac{v_{1}+v_{2}}{2}\right) & \leq \frac{\psi_{(x)}^{P} \mathrm{~J}_{\psi^{-1}\left(\nu_{1}\right)+}^{\eta_{1}, \eta_{2}, \gamma, \omega}(f \circ \psi)\left(\psi^{-1}\left(v_{2}\right)\right)+\underset{\psi(x)}{P} \mathrm{~J}_{\psi^{-1}\left(\nu_{2}\right)-}^{\eta_{1}, \eta_{2}, \gamma, \omega}(f \circ \psi)\left(\psi^{-1}\left(v_{1}\right)\right)}{2\left(v_{2}-v_{1}\right)^{\eta_{2}} E_{\eta_{1}, \eta_{2}+1}^{\gamma}\left(\omega\left(v_{2}-v_{1}\right)^{\eta_{1}}\right)} \\
& \leq \frac{f\left(v_{1}\right)+f\left(v_{2}\right)}{2}
\end{aligned}
$$

Proof As in the theorem proof, analogous to (22), we have the following key result due to [17]:

$$
\underset{\psi(x)}{P} \mathrm{~J}_{\psi^{-1}\left(\nu_{1}\right)+}^{\eta_{1}, \eta_{2}, \gamma, \omega} f(x)=\sum_{k=0}^{\infty} \frac{\Gamma(\gamma+k) \omega^{k}}{\Gamma(\gamma) k !} \underset{\psi(x)}{R L} \mathrm{~J}_{\psi^{-1}\left(\nu_{1}\right)+}^{k \eta_{1}+\eta_{2}} f(x) .
$$

Again, this series is locally uniformly convergent (see [17]).

By making use of $\mathrm{HH}$-inequality (18) and replacing $\eta_{1}$ by $k \eta_{1}+\eta_{2}$, we get

$$
\begin{aligned}
& f\left(\frac{v_{1}+v_{2}}{2}\right) \leq \frac{\Gamma\left(k \eta_{1}+\eta_{2}+1\right)}{2\left(v_{2}-v_{1}\right)^{k \eta_{1}+\eta_{2}}}\left[\begin{array}{c}
R L(x) \\
\mathrm{J}_{\psi^{-1}\left(\nu_{1}\right)+}^{k \eta_{1}+\eta_{2}}
\end{array}(f \circ \psi)\left(\psi^{-1}\left(v_{2}\right)\right)\right. \\
& \left.+\underset{\psi(x)}{R L} \mathrm{~J}_{\psi^{-1}\left(v_{2}\right)_{-}}^{k \eta_{1}+\eta_{2}}(f \circ \psi)\left(\psi^{-1}\left(v_{1}\right)\right)\right] \leq \frac{f\left(v_{1}\right)+f\left(v_{2}\right)}{2} .
\end{aligned}
$$


Since the multiplier in the central term is positive and $\gamma, \omega>0$ in series (27), so we can proceed as follows:

$$
\begin{aligned}
& \frac{\Gamma(\gamma+k) \omega+}{\Gamma(\gamma) k !} \cdot \frac{2\left(v_{2}-v_{1}\right)^{k \eta_{1}+\eta_{2}}}{\Gamma\left(k \eta_{1}+\eta_{2}+1\right)} f\left(\frac{v_{1}+v_{2}}{2}\right) \\
& \quad \leq \frac{\Gamma(\gamma+k) \omega+}{\Gamma(\gamma) k !}\left[\begin{array}{c}
R L \\
\psi(x)
\end{array} \mathrm{J}_{\psi^{-1}\left(\nu_{1}\right)+}^{k \eta_{1}+\eta_{2}}(f \circ \psi)\left(\psi^{-1}\left(v_{2}\right)\right)+\begin{array}{c}
R L \\
\psi(x) \\
\mathrm{J}^{k \eta^{-1}\left(\nu_{2}\right)-}
\end{array}(f \circ \psi)\left(\psi^{-1}\left(v_{1}\right)\right)\right] \\
& \quad \leq \frac{\Gamma(\gamma+k) \omega+}{\Gamma(\gamma) k !} \cdot \frac{2\left(v_{2}-v_{1}\right)^{k \eta_{1}+\eta_{2}}}{\Gamma\left(k \eta_{1}+\eta_{2}+1\right)} \cdot \frac{f\left(v_{1}\right)+f\left(v_{2}\right)}{2} .
\end{aligned}
$$

Summing over all $k$ to get

$$
\begin{aligned}
\sum_{k=0}^{\infty} & \frac{\Gamma(\gamma+k) \omega+}{\Gamma(\gamma) k !} \cdot \frac{2\left(v_{2}-v_{1}\right)^{k \eta_{1}+\eta_{2}}}{\Gamma\left(k \eta_{1}+\eta_{2}+1\right)} f\left(\frac{v_{1}+v_{2}}{2}\right) \\
& \leq \underset{\psi(x)}{P} \mathrm{~J}_{\psi^{-1}\left(\nu_{1}, \eta_{1}, \gamma^{\prime}, \omega\right.}(f \circ \psi)\left(\psi^{-1}\left(v_{2}\right)\right)+\underset{\psi(x)}{P} \mathrm{~J}_{\psi^{-1}\left(v_{2}\right)-}^{\eta_{1}, \eta_{2}, \gamma, \omega}(f \circ \psi)\left(\psi^{-1}\left(v_{1}\right)\right) \\
& \leq \sum_{k=0}^{\infty} \frac{\Gamma(\gamma+k) \omega+}{\Gamma(\gamma) k !} \cdot \frac{2\left(v_{2}-v_{1}\right)^{k \eta_{1}+\eta_{2}}}{\Gamma\left(k \eta_{1}+\eta_{2}+1\right)} \cdot \frac{f\left(v_{1}\right)+f\left(v_{2}\right)}{2} .
\end{aligned}
$$

The left- and right-hand sides series in this inequality can be rewritten as the power series for the three-parameter ML function:

$$
\begin{aligned}
& 2\left(v_{2}-v_{1}\right)^{\eta_{2}} E_{\eta_{1}, \eta_{2}+1}^{\gamma}\left(\omega\left(v_{2}-v_{1}\right)^{\eta_{1}}\right) f\left(\frac{v_{1}+v_{2}}{2}\right) \\
& \leq \underset{\psi(x)}{P} \mathrm{~J}_{\psi^{-1}\left(v_{1}\right)_{+}}^{\eta_{1}, \eta_{2}, \gamma, \omega}(f \circ \psi)\left(\psi^{-1}\left(v_{2}\right)\right)+\underset{\psi(x)}{P} \mathrm{~J}_{\psi^{-1}\left(v_{2}\right)-}^{\eta_{1}, \eta_{2}, \gamma, \omega}(f \circ \psi)\left(\psi^{-1}\left(v_{1}\right)\right) \\
& \leq 2\left(v_{2}-v_{1}\right)^{\eta_{2}} E_{\eta_{1}, \eta_{2}+1}^{\gamma}\left(\omega\left(v_{2}-v_{1}\right)^{\eta_{1}}\right) \frac{f\left(v_{1}\right)+f\left(v_{2}\right)}{2} .
\end{aligned}
$$

The result follows by a simple rearrangement.

Corollary 3.3 Theorem 3.3 with $\psi(x)=x$ becomes

$$
f\left(\frac{\nu_{1}+v_{2}}{2}\right) \leq \frac{{ }^{P} \mathrm{~J}_{\nu_{1}}^{\eta_{1}, \eta_{2}, \gamma, \omega} f\left(v_{2}\right)+{ }^{P} \mathrm{~J}_{\nu_{2}}^{\eta_{1}, \eta_{2}, \gamma, \omega} f\left(v_{1}\right)}{2\left(v_{2}-v_{1}\right)^{\eta_{2}} E_{\eta_{1}, \eta_{2}+1}^{\gamma}\left(\omega\left(v_{2}-v_{1}\right)^{\left.\eta_{1}\right)}\right.} \leq \frac{f\left(v_{1}\right)+f\left(v_{2}\right)}{2},
$$

which is proved by Fernandez and Mohammed in [19, Theorem 2.2].

Example 3.2 We verify our result in Theorem 3.3 for $\psi(x)=x$ and the convex function $f(x)=e^{2 x}$ on the interval $[0,1]$. By making use of (24) and (27), it follows that

$$
\begin{aligned}
& P_{\mathrm{J}_{d+}^{\eta_{1}, \eta_{2}, \gamma, \omega}} e^{2 x}=e^{2 x} \sum_{k=0}^{\infty} \frac{\Gamma(\gamma+k) \omega+}{\Gamma(\gamma) k !} 2^{-k \eta_{1}-\eta_{2}} \frac{\gamma\left(k \eta_{1}+\eta_{2}, 2(x-d)\right)}{\Gamma\left(k \eta_{1}+\eta_{2}\right)}, \\
& { }^{P} \mathrm{~J}_{d-}^{\eta_{1}, \eta_{2}, \gamma, \omega} e^{2 x}=e^{2 x} \sum_{k=0}^{\infty} \frac{\Gamma(\gamma+k) \omega+}{\Gamma(\gamma) k !}(-2)^{-k \eta_{1}-\eta_{2}} \frac{\gamma\left(k \eta_{1}+\eta_{2}, 2(x-d)\right)}{\Gamma\left(k \eta_{1}+\eta_{2}\right)} .
\end{aligned}
$$




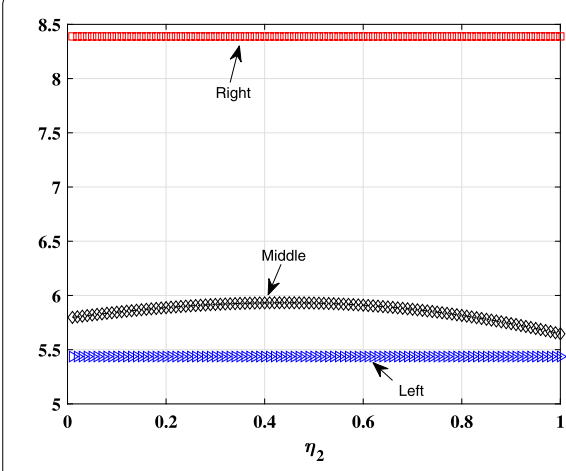

(a) For $\eta_{1}=\frac{1}{2}, \gamma=4$

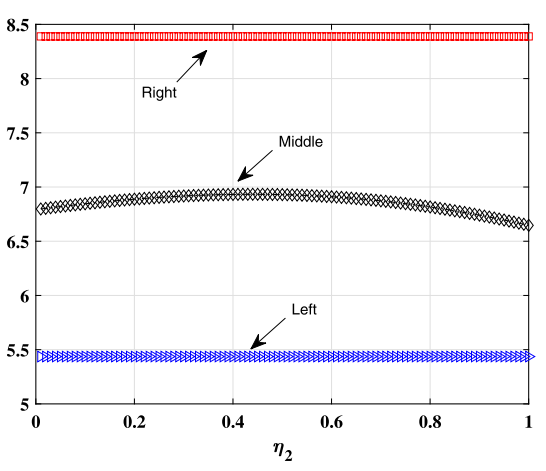

(b) For $\eta_{1}=\frac{1}{4}, \gamma=2$

Figure 2 Plot illustrations for Example 3.2

Substituting the above expressions into $\mathrm{HH}$-inequality (26), we get

$$
\begin{aligned}
e & \leq \frac{e^{2} \sum_{k=0}^{\infty} \frac{\Gamma(\gamma+k) \omega+}{\Gamma(\gamma) k !} 2^{-k \eta_{1}-\eta_{2}} \frac{\gamma\left(k\left(\eta_{1}+\eta_{2}, 2\right)\right.}{\Gamma\left(k \eta_{1}+\eta_{2}\right)}+\sum_{k=0}^{\infty} \frac{\Gamma(\gamma+k) \omega+}{\Gamma(\gamma) k !}(-2)^{-k \eta_{1}-\eta_{2}} \frac{\gamma\left(k \eta_{1}+\eta_{2},-2\right)}{\Gamma\left(k \eta_{1}+\eta_{2}\right)}}{2 E_{\eta_{1}, \eta_{2}+1}^{\gamma}(\omega)} \\
& \leq \frac{1+e^{2}}{2}
\end{aligned}
$$

which may be simplified to

$$
\begin{aligned}
2 e \leq & \frac{1}{\Gamma(\gamma) E_{\eta_{1}, \eta_{2}+1}^{\gamma}(\omega)} \sum_{k=0}^{\infty} \frac{\Gamma(\gamma+k) \omega+}{k ! \Gamma\left(k \eta_{1}+\eta_{2}\right)}\left[e^{2} 2^{-k \eta_{1}-\eta_{2}} \gamma\left(k \eta_{1}+\eta_{2}, 2\right)\right. \\
& \left.+(-2)^{-k \eta_{1}-\eta_{2}} \gamma\left(k \eta_{1}+\eta_{2},-2\right)\right] \leq 1+e^{2}
\end{aligned}
$$

For further illustration of this result, we set $\omega=1$ and allow $\eta_{2}$ to vary between 0 and 1 . Also, for several choices of $\eta_{1}$ and $\gamma$, the results are shown in Fig. 2. In each situation, the middle inequality of (28) is between the constant values of the left- and right-hand sides.

\section{A related integral equality}

Hermite-Hadamard ( $\mathrm{HH})$ inequalities are strongly connected with further well-known integral inequalities which are said to be of trapezoidal type in the literature, see e.g. [11, $36-42,47,53,54]$. In the coming theorem, we prepare an equality of trapezoidal type for the new integral operator. Also, we believe this result will be vital information for the future studies of these models of fractional calculus.

Theorem 4.1 Suppose that $f:\left[v_{1}, v_{2}\right] \rightarrow \mathfrak{R}$ is an $\mathrm{L}^{1}$ function with $\eta_{1} \in(0,1)$. Also, suppose that $f^{\prime} \in \mathrm{L}^{1}\left(\left[v_{1}, v_{2}\right)\right]$, and $\psi$ is an increasing positive function on $\left(v_{1}, v_{2}\right]$ with $\psi^{\prime}(x) \in$ $\mathrm{L}^{1}\left(v_{1}, v_{2}\right)$, then we have

$$
\begin{aligned}
& \frac{f\left(v_{1}\right)+f\left(v_{2}\right)}{2}-\frac{B\left(\eta_{1}\right) \Gamma\left(\eta_{1}\right)}{2\left[\left(v_{2}-v_{1}\right)^{\eta_{1}}+\left(1-\eta_{1}\right) \Gamma\left(\eta_{1}\right)\right]}\left[\underset{\psi(x)}{A B} \widetilde{J}_{\psi^{-1}\left(v_{1}\right)+}^{\eta_{1}}(f \circ \psi)\left(\psi^{-1}\left(v_{2}\right)\right)\right. \\
& \left.+\underset{\psi(x)}{A B} \mathrm{~J}_{\psi^{-1}\left(v_{2}\right)-}^{\eta_{1}}(f \circ \psi)\left(\psi^{-1}\left(v_{1}\right)\right)\right]
\end{aligned}
$$




$$
\begin{aligned}
= & \frac{\left(v_{2}-v_{1}\right)^{\eta_{1}-1}}{2\left[\left(v_{2}-v_{1}\right)^{\eta_{1}}+\left(1-\eta_{1}\right) \Gamma\left(\eta_{1}\right)\right]} \\
& \times \int_{\psi^{-1}\left(v_{1}\right)}^{\psi^{-1}\left(v_{2}\right)}\left[\left(\psi(\xi)-v_{1}\right)^{\eta_{1}}-\left(v_{2}-\psi(\xi)\right)^{\eta_{1}}\right]\left(f^{\prime} \circ \psi\right)(\xi) \psi^{\prime}(\xi) \mathrm{d} \xi
\end{aligned}
$$

Proof By making use of Lemma 3.1, we get

$$
\begin{aligned}
& \underset{\psi(x)}{R L} \mathrm{~J}_{\psi^{-1}\left(v_{1}\right)_{+}}^{\eta_{1}}(f \circ \psi)\left(\psi^{-1}\left(v_{2}\right)\right)=\frac{B\left(\eta_{1}\right)}{\eta_{1}} \underset{\psi(x)}{A B} \mathrm{~J}_{\psi^{-1}\left(v_{1}\right)_{+}}^{\eta_{1}}(f \circ \psi)\left(\psi^{-1}\left(v_{2}\right)\right)-\frac{1-\eta_{1}}{\eta_{1}} f\left(v_{2}\right) ; \\
& \underset{\psi(x)}{R L} \mathrm{~J}_{\psi^{-1}\left(v_{2}\right)_{-}}^{\eta_{1}}(f \circ \psi)\left(\psi^{-1}\left(v_{1}\right)\right)=\frac{B\left(\eta_{1}\right)}{\eta_{1}} \underset{\psi(x)}{A B} \mathrm{~J}_{\psi^{-1}\left(v_{2}\right)_{-}}^{\eta_{1}}(f \circ \psi)\left(\psi^{-1}\left(v_{1}\right)\right)-\frac{1-\eta_{1}}{\eta_{1}} f\left(v_{1}\right) .
\end{aligned}
$$

By adding the last two inequalities together and then multiplying by $\frac{\Gamma\left(\eta_{1}+1\right)}{2\left(v_{2}-v_{1}\right)^{\eta_{1}}}>0$, we obtain

$$
\begin{aligned}
& \frac{\Gamma\left(\eta_{1}+1\right)}{2\left(v_{2}-v_{1}\right)^{\eta_{1}}}\left[\underset{\psi(x)}{R L} \mathrm{~J}_{\psi^{-1}\left(v_{1}\right)+}^{\eta_{1}} f\left(v_{2}\right)+\underset{\psi(x)}{R L} \mathrm{~J}_{\psi^{-1}\left(v_{2}\right)}^{\eta_{1}} f\left(v_{1}\right)\right] \\
& =\frac{B\left(\eta_{1}\right) \Gamma\left(\eta_{1}\right)}{2\left(v_{2}-v_{1}\right)^{\eta_{1}}}\left[\underset{\psi(x)}{A B} \mathrm{~J}_{\psi^{-1}\left(v_{1}\right)+}^{\eta_{1}}(f \circ \psi)\left(\psi^{-1}\left(v_{2}\right)\right)+\underset{\psi(x)}{A B} \mathrm{~J}_{\psi^{-1}\left(v_{2}\right)-}^{\eta_{1}}(f \circ \psi)\left(\psi^{-1}\left(v_{1}\right)\right)\right] \\
& \quad-\frac{\left(1-\eta_{1}\right) \Gamma\left(\eta_{1}\right)}{\left(v_{2}-v_{1}\right)^{\eta_{1}}} \cdot \frac{f\left(v_{1}\right)+f\left(v_{2}\right)}{2} .
\end{aligned}
$$

This gives

$$
\begin{aligned}
& \frac{f\left(v_{1}\right)+f\left(v_{2}\right)}{2}-\frac{\Gamma\left(\eta_{1}+1\right)}{2\left(v_{2}-v_{1}\right)^{\eta_{1}}}\left[\underset{\psi(x)}{R L} \mathrm{~J}_{\psi^{-1}\left(v_{1}\right)}^{\eta_{1}} f\left(v_{2}\right)+\underset{\psi(x)}{R L} \mathrm{~J}_{\psi^{-1}\left(v_{2}\right)}^{\eta_{1}} f\left(v_{1}\right)\right] \\
& =\frac{\left(\nu_{2}-v_{1}\right)^{\eta_{1}}+\left(1-\eta_{1}\right) \Gamma\left(\eta_{1}\right)}{\left(v_{2}-v_{1}\right)^{\eta_{1}}} \cdot \frac{f\left(v_{1}\right)+f\left(\nu_{2}\right)}{2}-\frac{B\left(\eta_{1}\right) \Gamma\left(\eta_{1}\right)}{2\left(\nu_{2}-v_{1}\right)^{\eta_{1}}} \\
& \times\left[\underset{\psi(x)}{A B} \mathrm{~J}_{\psi^{-1}\left(\nu_{1}\right)+}^{\eta_{1}}(f \circ \psi)\left(\psi^{-1}\left(v_{2}\right)\right)+\underset{\psi(x)}{A B} \mathrm{~J}_{\psi^{-1}\left(\nu_{2}\right)-}^{\eta_{1}}(f \circ \psi)\left(\psi^{-1}\left(v_{1}\right)\right)\right] .
\end{aligned}
$$

Following [30, Lemma 3.1], it follows that

$$
\begin{aligned}
& \frac{f\left(v_{1}\right)+f\left(v_{2}\right)}{2}-\frac{\Gamma\left(\eta_{1}+1\right)}{2\left(v_{2}-v_{1}\right)^{\eta_{1}}}\left[\begin{array}{c}
R L(x) \\
\psi
\end{array} 丁_{\psi^{-1}\left(v_{1}\right)+}^{\eta_{1}}(f \circ \psi)\left(\psi^{-1}\left(v_{2}\right)\right)\right. \\
& \left.\quad+\underset{\psi(x)}{R L} \mathrm{~J}_{\psi^{-1}\left(v_{2}\right)-}^{\eta_{1}}(f \circ \psi)\left(\psi^{-1}\left(v_{1}\right)\right)\right] \\
& =\frac{1}{2\left(v_{2}-v_{1}\right)} \int_{\psi^{-1}\left(v_{1}\right)}^{\psi^{-1}\left(v_{2}\right)}\left[\left(\psi(\xi)-v_{1}\right)^{\eta_{1}}-\left(v_{2}-\psi(\xi)\right)^{\eta_{1}}\right]\left(f^{\prime} \circ \psi\right)(\xi) \psi^{\prime}(\xi) \mathrm{d} \xi .
\end{aligned}
$$

Making use of equations (30) and (31) completes the proof.

Corollary 4.1 Theorem 4.1 with $\psi(x)=x$ becomes

$$
\begin{aligned}
& \frac{f\left(v_{1}\right)+f\left(v_{2}\right)}{2}-\frac{B\left(\eta_{1}\right) \Gamma\left(\eta_{1}\right)}{2\left[\left(v_{2}-v_{1}\right)^{\eta_{1}}+\left(1-\eta_{1}\right) \Gamma\left(\eta_{1}\right)\right]}\left[{ }^{A B} \mathrm{~J}_{v_{1}+}^{\eta_{1}} f\left(v_{2}\right)+{ }^{A B} \mathrm{~J}_{v_{2}-}^{\eta_{1}} f\left(v_{1}\right)\right] \\
& =\frac{\left(v_{2}-v_{1}\right)^{\eta_{1}+1}}{2\left[\left(v_{2}-v_{1}\right)^{\eta_{1}}+\left(1-\eta_{1}\right) \Gamma\left(\eta_{1}\right)\right]} \int_{0}^{1}\left[(1-\xi)^{\eta_{1}}-\xi^{\eta_{1}}\right] f^{\prime}\left(t v_{1}+(1-t) v_{2}\right) \mathrm{d} \xi
\end{aligned}
$$

which is proved by Fernandez and Mohammed in [19, Theorem 3.1]. 


\section{Conclusions}

In this article, we have performed a connection between the Atangana-Baleanu and Riemann-Liouville fractional integrals of a function with respect to an increasing function with nonsingular kernel. Also, we have recalled the iterated formula of the Prabhakar fractional operator implemented by Fernandez and Baleanu [17]. In view of these indices, we have presented new efficient inequalities of Hermite-Hadamard type (HH-type) in the context of fractional calculus with respect to functions involving nonsingular kernels. In fact, our findings generalize those in [19]. Due to the extensive recent studies and applications of $\mathrm{AB}$ and Prabhakar fractional operators, we believe that our results will be important for the future studies of those models of fractional calculus and integral inequality.

\section{Acknowledgements}

The second author would like to thank Prince Sultan University for funding this work through research group Nonlinear Analysis Methods in Applied Mathematics (NAMAM), group number RG-DES-2017-01-17.

Funding

Not applicable.

Availability of data and materials

Not applicable.

Competing interests

The authors declare that they have no competing interests.

Consent for publication

Not applicable.

Authors' contributions

All authors contributed equally and significantly in writing this article. All authors read and approved the final manuscript.

\section{Author details}

'Department of Mathematics, College of Education, University of Sulaimani, Sulaimani, Kurdistan Region, Iraq.

${ }^{2}$ Department of Mathematics and General Sciences, Prince Sultan University, P.O. Box 66833, Riyadh 11586, Saudi Arabia.

${ }^{3}$ Department of Medical Research, China Medical University, Taichung 40402, Taiwan. ${ }^{4}$ Department of Computer Science and Information Engineering, Asia University, Taichung, Taiwan.

\section{Publisher's Note}

Springer Nature remains neutral with regard to jurisdictional claims in published maps and institutional affiliations.

Received: 4 April 2020 Accepted: 7 July 2020 Published online: 16 July 2020

\section{References}

1. Abdeljawad, T.: A Lyapunov type inequality for fractional operators with nonsingular Mittag-Leffler kernel. J. Inequal. Appl. 2017, $130(2017)$

2. Abdeljawad, T:. Fractional operators with generalized Mittag-Leffler kernels and their differintegrals. Chaos Solitons Fractals 29, 023102 (2019)

3. Abdeljawad, T.: Fractional difference operators with discrete generalized Mittag-Leffler kernels. Chaos Solitons Fractals 126, 315-324 (2019)

4. Abdeljawad, T., Al-Mdallal, Q.M.: Discrete Mittag-Leffler kernel type fractional difference initial value problems and Gronwall's inequality. J. Comput. Appl. Math. 339, 218-230 (2018)

5. Abdeljawad, T., Baleanu, D.: Integration by parts and its applications of a new nonlocal fractional derivative with Mittag-Leffler nonsingular kernel. J. Nonlinear Sci. Appl. 10(3), 1098-1107 (2017)

6. Acay, B., Bas, E., Abdeljawad, T.: Fractional economic models based on market equilibrium in the frame of different type kernels. Chaos Solitons Fractals 130, 109438 (2020)

7. Anastassiou, G.A.: Opial type inequalities involving Riemann-Liouville fractional derivatives of two functions with applications. Math. Comput. Model. 48, 344-374 (2008)

8. Atangana, A.: Fractional Operators with Constant and Variable Order with Application to Geo-hydrology. Academic Press, New York (2017)

9. Atangana, A., Baleanu, D.: New fractional derivatives with nonlocal and non-singular kernel: theory and application to heat transfer model. Therm. Sci. 20(2), 763-769 (2016)

10. Baleanu, D., Fernandez, A.: On some new properties of fractional derivatives with Mittag-Leffler kernel. Commun. Nonlinear Sci. Numer. Simul. 59, 444-462 (2018)

11. Baleanu, D., Mohammed, P.O., Zeng, S.: Inequalities of trapezoidal type involving generalized fractional integrals. Alex. Eng. J. https://doi.org/10.1016/j.aej.2020.03.039 
12. Baleanu, D., Shiri, B.: Collocation methods for fractional differential equations involving non-singular kernel. Chaos Solitons Fractals 116, 136-145 (2018)

13. Baleanu, D., Shiri, B., Srivastava, H.M., Al Qurashi, M.: A Chebyshev spectral method based on operational matrix for fractional differential equations involving non-singular Mittag-Leffler kernel. Adv. Differ. Equ. 2018, 353 (2018)

14. Bonyah, E., Atangana, A., Elsadany, A.A.: A fractional model for predator-prey with omnivore. Chaos 29, 013136 (2019)

15. Djida, J.-D., Atangana, A., Area, I.: Numerical computation of a fractional derivative with non-local and non-singular kernel. Math. Model. Nat. Phenom. 12(3), 4-13 (2017)

16. Dragomir, S.S., Bhatti, M.I., lqbal, M., Muddassar, M.: Some new Hermite-Hadamard's type fractional integral inequalities. J. Comput. Anal. Appl. 18(4), 655-661 (2015)

17. Fernandez, A., Baleanu, D.: Differintegration with respect to functions in fractional models involving Mittag-Leffler functions. SSRN Electron. J. (2018). https://doi.org/10.2139/ssrn.3275746

18. Fernandez, A., Baleanu, D., Srivastava, H.M.: Series representations for models of fractional calculus involving generalised Mittag-Leffler functions. Commun. Nonlinear Sci. Numer. Simul. 67, 517-527 (2019)

19. Fernandez, A., Mohammed, P.O.: Hermite-Hadamard inequalities in fractional calculus defined using Mittag-Leffler kernels. Math. Methods Appl. Sci., 1-18 (2020). https://doi.org/10.1002/mma.6188

20. Garra, R., Garrappa, R.: The Prabhakar or three parameter Mittag-Leffler function: theory and application. Commun. Nonlinear Sci. Numer. Simul. 56, 314-329 (2018)

21. Gorenflo, R., Kilbas, A.A., Mainardi, F., Rogosin, V.: Mittag-Leffler Functions, Related Topics and Applications. Springer, Berlin (2014)

22. Haubold, H.J., Mathai, A.M., Saxena, R.K.: Mittag-Leffler functions and their applications. J. Appl. Math. 2011, Article ID 298628 (2011)

23. Hristov, J.: The Craft of Fractional Modelling in Science and Engineering. MDPI, Basel (2018)

24. Jarad, F., Abdeljawad, T., Hammouch, Z.: On a class of ordinary differential equations in the frame of Atangana-Baleanu fractional derivative. Chaos Solitons Fractals 117, 16-20 (2018)

25. Kavurmaci, H., Avci, M., Ozdemir, M.E.: New inequalities of Hermite-Hadamard type for convex functions with applications. J. Inequal. Appl. 2011, 86 (2011)

26. Khan, H., Abdeljawad, T., Tunc, C., Alkhazzan, A., Khan, A.: Minkowskis inequality for the AB-fractional integral operator. J. Inequal. Appl. 2019, 96 (2019)

27. Kilbas, A.A., Saigo, M., Saxena, R.K.: Generalized Mittag-Leffler function and generalized fractional calculus operators. Integral Transforms Spec. Funct. 15(1), 31-49 (2004)

28. Kilbas, A.A., Srivastava, H.M., Trujillo, J.J.: Theory and Applications of Fractional Differential Equations. Elsevier, Amsterdam (2006)

29. Lakshmikantham, V., Leela, S.: Differential and Integral Inequalities: Theory and Applications: Volume I: Ordinary Differential Equations. Academic Press, New York (1969)

30. Liu, K., Wang, J., O'Regan, D.: On the Hermite-Hadamard type inequality for $\psi$-Riemann-Liouville fractional integrals via convex functions. J. Inequal. Appl. 2019, 27 (2019)

31. Mathai, A.M., Haubold, H.J.: Mittag-Leffler functions and fractional calculus. In: Special Functions for Applied Scientists. Springer, New York (2008)

32. Miller, K.S., Ross, B.: An Introduction to the Fractional Calculus and Fractional Differential Equations. Wiley, New York (1993)

33. Mohammed, P.O.: Some integral inequalities of fractional quantum type. Malaya J. Mat. 4(1), 93-99 (2016)

34. Mohammed, P.O.: Some new Hermite-Hadamard type inequalities for MT-convex functions on differentiable coordinates. J. King Saud Univ., Sci. 30(2), 258-262 (2018)

35. Mohammed, P.O.: A generalized uncertain fractional forward difference equations of Riemann-Liouville type. J. Math Res. 11(4), 43-50 (2019)

36. Mohammed, P.O.: Hermite-Hadamard inequalities for Riemann-Liouville fractional integrals of a convex function with respect to a monotone function. Math. Methods Appl. Sci., 1-11 (2019). https://doi.org/10.1002/mma.5784

37. Mohammed, P.O., Abdeljawad, T.: Modification of certain fractional integral inequalities for convex functions. Adv. Differ. Equ. 2020, 69 (2020)

38. Mohammed, P.O., Brevik, I.: A new version of the Hermite-Hadamard inequality for Riemann-Liouville fractional integrals. Symmetry 12, 610 (2020). https://doi.org/10.3390/sym12040610

39. Mohammed, P.O., Hamasalh, F.K.: New conformable fractional integral inequalities of Hermite-Hadamard type for convex functions. Symmetry 11(2), 263 (2019). https://doi.org/10.3390/sym11020263

40. Mohammed, P.O., Sarikaya, M.Z.: Hermite-Hadamard type inequalities for F-convex function involving fractional integrals. J. Inequal. Appl. 2018, 359 (2018)

41. Mohammed, P.O., Sarikaya, M.Z.: On generalized fractional integral inequalities for twice differentiable convex functions. J. Comput. Appl. Math. 372, 112740 (2020)

42. Mohammed, P.O., Sarikaya, M.Z., Baleanu, D.: On the generalized Hermite-Hadamard inequalities via the tempered fractional integrals. Symmetry 12, 595 (2020). https://doi.org/10.3390/sym12040595

43. Oldham, K.B., Spanier, J.: The Fractional Calculus. Academic Press, San Diego (1974)

44. Osler, T.J.: Fractional derivatives of a composite function. SIAM J. Math. Anal. 1, 288-293 (1970)

45. Pollard, H.: The completely monotonic character of the Mittag-Leffler function $E_{\alpha}(-x)$. Bull. Am. Math. Soc. 54(12), $1115-1116(1948)$

46. Prabhakar, T.R.: A singular integral equation with a generalized Mittag Leffler function in the kernel. Yokohama Math. J. 19, 7-15 (1971)

47. Qi, F., Mohammed, P.O., Yao, J.C., Yao, Y.H.: Generalized fractional integral inequalities of Hermite-Hadamard type for $(\alpha, m)$-convex functions. J. Inequal. Appl. 2019, 135 (2019)

48. Rashid, S., Abdeljawad, T., Jarad, F., Noor, M.A.: Some estimates for generalized Riemann-Liouville fractional integrals of exponentially convex functions and their applications. Mathematics 7, 807 (2019)

49. Rashid, S., Jarad, F., Noor, M.A., Noor, K.I., Baleanu, D., Liu, J.-B.: On Grüss inequalities within generalized K-fractional integrals. Adv. Differ. Equ. 2020, 203 (2020) 
50. Rashid, S., Kalsoom, H., Hammouch, Z., Ashraf, R., Baleanu, D., Chu, Y.-M.: New multi-parametrized estimates having pth-order differentiability in fractional calculus for predominating $h$-convex functions in Hilbert space. Symmetry 12 , $222(2020)$

51. Rashid, S., Noor, M.A., Noor, K.I., Chu, Y.-M.: Ostrowski type inequalities in the sense of generalized K-fractional integral operator for exponentially convex functions. AIMS Math. 5(3), 2629-2645 (2020)

52. Samko, S.G., Kilbas, A.A., Marichev, O.I.: Fractional Integrals and Derivatives: Theory and Applications. Taylor \& Francis, London (2002) [orig. ed. in Russian, Nauka i Tekhnika, Minsk, 1987]

53. Sarikaya, M.Z., Set, E., Yaldiz, H., Başak, N.: Hermite-Hadamard's inequalities for fractional integrals and related fractional inequalities. Math. Comput. Model. 57, 2403-2407 (2013)

54. Sarikaya, M.Z., Yildirim, H.: On Hermite-Hadamard type inequalities for Riemann-Liouville fractional integrals. Miskolc Math. Notes 17(2), 1049-1059 (2017)

55. Shiri, B., Baleanu, D.: System of fractional differential algebraic equations with applications. Chaos Solitons Fractals 120, 203-212 (2019)

56. Sousa, J.V.C., Oliveira, E.C.: On the $\Psi$-Hilfer fractional derivative. Commun. Nonlinear Sci. Numer. Simul. 60, 72-91 (2018)

57. Srivastava, H.M., Tomovski, Z.: Fractional calculus with an integral operator containing a generalized Mittag-Leffler function in the kernel. Appl. Math. Comput. 211(1), 198-210 (2009)

58. Uçar, S., Uçar, E., Özdemir, N., Hammouch, Z.: Mathematical analysis and numerical simulation for a smoking model with Atangana-Baleanu derivative. Chaos Solitons Fractals 118, 300-306 (2019)

59. Walter, W.: Differential and Integral Inequalities, vol. 55. Springer, Berlin (2012) [orig. ed. in German; Springer Tracts in Natural Philosophy, 1964]

\section{Submit your manuscript to a SpringerOpen ${ }^{\circ}$ journal and benefit from:}

- Convenient online submission

- Rigorous peer review

Open access: articles freely available online

- High visibility within the field

- Retaining the copyright to your article

Submit your next manuscript at $>$ springeropen.com 